\title{
Bioengineered Polyhydroxyalkanoates as Immobilized Enzyme Scaffolds for Industrial Applications
}

\author{
Jin Xiang Wong',2, Kampachiro Ogura ${ }^{1}$, Shuxiong Chen ${ }^{3}$ and Bernd H. A. Rehm ${ }^{3,4 *}$ \\ ${ }^{1}$ School of Fundamental Sciences, Massey University, Palmerston North, New Zealand, ${ }^{2}$ MacDiarmid Institute of Advanced \\ Materials and Nanotechnology, Victoria University of Wellington, Wellington, New Zealand, ${ }^{3}$ Centre for Cell Factories \\ and Biopolymers, Griffith Institute for Drug Discovery, Griffith University, Nathan, QLD, Australia, ${ }^{4}$ Menzies Health Institute \\ Queensland (MHIQ), Griffith University, Gold Coast Campus, Southport, QLD, Australia
}

\section{OPEN ACCESS}

Edited by:

Spiros Nicolas Agathos,

Catholic University of Louvain,

Belgium

Reviewed by:

Abdelmageed M. Othman, National Research Centre (Egypt),

Egypt

Elena Dimitrova Vassileva,

Sofia University, Bulgaria

*Correspondence:

Bernd H. A. Rehm b.rehm@griffith.edu.au

Specialty section: This article was submitted to

Industrial Biotechnology,

a section of the journal Frontiers in Bioengineering and Biotechnology

Received: 20 September 2019 Accepted: 14 February 2020 Published: 04 March 2020

Citation:

Wong JX, Ogura K, Chen S and Rehm BHA (2020) Bioengineered

Polyhydroxyalkanoates as Immobilized Enzyme Scaffolds

for Industrial Applications.

Front. Bioeng. Biotechnol. 8:156.

doi: 10.3389/fbioe.2020.00156
Enzymes function as biocatalysts and are extensively exploited in industrial applications. Immobilization of enzymes using support materials has been shown to improve enzyme properties, including stability and functionality in extreme conditions and recyclability in biocatalytic processing. This review focuses on the recent advances utilizing the design space of in vivo self-assembled polyhydroxyalkanoate (PHA) particles as biocatalyst immobilization scaffolds. Self-assembly of biologically active enzyme-coated PHA particles is a one-step in vivo production process, which avoids the costly and laborious in vitro chemical cross-linking of purified enzymes to separately produced support materials. The homogeneous orientation of enzymes densely coating PHA particles enhances the accessibility of catalytic sites, improving enzyme function. The PHA particle technology has been developed into a remarkable scaffolding platform for the design of cost-effective designer biocatalysts amenable toward robust industrial bioprocessing. In this review, the PHA particle technology will be compared to other biological supramolecular assembly-based technologies suitable for in vivo enzyme immobilization. Recent progress in the fabrication of biological particulate scaffolds using enzymes of industrial interest will be summarized. Additionally, we outline innovative approaches to overcome limitations of in vivo assembled PHA particles to enable finetuned immobilization of multiple enzymes to enhance performance in multi-step cascade reactions, such as those used in continuous flow bioprocessing.

Keywords: polyhydroxyalkanoate, enzyme immobilization, synthetic biology, industrial biotechnology, polymers

\section{ENZYME IMMOBILIZATION FOR INDUSTRIAL APPLICATIONS}

Enzymes are capable of accelerating chemical reactions with high substrate specificity, stereoselectivity, and energy-efficient conversion properties (Robinson, 2015). These enzyme properties attract interest from the biotechnology sector and are considered as a substitute to chemical catalysts in various applications, such as biomass conversion, food processing, and the production of pharmaceuticals (Chapman et al., 2018). Despite the excellent catalytic properties of enzymes, utilization of natural enzymes at industrial scales is often hampered by their general protein characteristics (Mohamad et al., 2015). For example, enzymes are prone to 
denaturation/unfolding when removed from their native environments. In particular, enzymes are sensitive to changes in their environments and are poorly stable in extreme conditions, such as high temperatures, high pressures, extreme $\mathrm{pHs}$, detergents, and organic solvents (Robinson, 2015). Furthermore, the complex reaction mixture makes it challenging to separate soluble enzymes and their respective products. Hence, enzymes are often rendered inactive and removed after a single use (Robinson, 2015). From an economic point of view, the poor reusability and difficulty in the recovery of enzymes are drawbacks, which severely limit the use of enzymes in industrial processes.

To overcome the shortcomings mentioned above, various enzyme immobilization techniques, especially scaffolding-based approaches, have been developed in the past decades (Cipolatti et al., 2017; Ren et al., 2019). Immobilization of enzymes results in the confinement of enzymes to a particular space, such as either displayed on, or encapsulated within, solid support materials, creating a heterogeneous biocatalyst system while retaining enzyme specificity and activity (Hartmeier, 2012). Interestingly, densely localizing enzymes on the scaffolding carriers can significantly improve enzymes' catalytic performance and structural stability in certain scenarios due to macromolecular crowding (Yang et al., 2017; Zaak et al., 2017). The non-specific interactions between the immobilized enzymes and solid support materials could also further enhance the overall function and stability of immobilized enzymes (Fu et al., 2012; Rodrigues et al., 2013; Jia et al., 2017). The crowding of globular proteins could also create an artificial environment that can improve protein stability against chaotropic agents and temperature stress (Minton, 2000).

Immobilized enzyme-based catalytic systems facilitate separation of the enzyme from the reaction mixture. This strategy enables the repeated use of the immobilized enzymes and rapid termination of a catalytic reaction by physically removing the immobilized enzyme-bearing carriers from the reaction mixture (Mohamad et al., 2015; Bernal et al., 2018). This approach also prevents contamination of the product by the carried-over enzyme, thus reducing downstream process complexity and operational costs. Moreover, immobilized enzyme-based biocatalysts allow the implementation of flowthrough formats in continuous bioprocessing approaches (Homaei et al., 2013; Zdarta et al., 2018). Nevertheless, in some cases, enzyme immobilization can impair the functionality of enzymes, as a result of unfavorable conformational changes in enzymes and restricted substrate access in comparison to their soluble counterpart (Guisan, 2006; Cao et al., 2012; Fernandez-Lopez et al., 2017). However, the advantages of enzyme immobilization outweigh their unfavorable impact and enhance the efficient implementation of biocatalysts in industrial processes.

Therefore, it is paramount to develop cost-effective and pragmatic enzyme immobilization approaches for potential industrial applications (Rehm et al., 2016, 2018; Nguyen and Kim, 2017). In general, scaffolding-based enzyme immobilization strategies can be categorized into in vitro and in vivo approaches. The in vitro approaches can offer excellent controllability by tuning the physicochemical properties of carriers (e.g., particle size and distribution, or surface charge) as well as by controlling the density of the immobilized enzymes (Faccio, 2018; GonzalezMiro et al., 2019). However, the in vitro methods often require harsh reaction conditions, such as the presence of toxic crosslinking agents, solvents, extreme temperatures, and $\mathrm{pHs,} \mathrm{for}$ successful enzyme immobilization, and these conditions can potentially compromise enzyme function (Sletten and Bertozzi, 2009). Furthermore, most in vitro immobilization methods (e.g., chemical modifications and physical adsorption) are not able to control the enzyme orientation on the solid supports, which directly influences the accessibility of substrates to the catalytic sites of enzymes (Hess et al., 2012; Brune et al., 2016). Also, due to the inherent structural complexity of the enzymes, localizing them onto support materials using existing in vitro conjugation technologies often necessitates labor-intensive reactions and process optimization steps (Krauss et al., 2017; Rehm et al., 2018). In addition, multiple separate manufacturing schemes are necessary for large-scale manufacturing of biocatalysts using in vitro cross-linking technologies (e.g., manufacturing lines for both enzyme and support materials, and subsequent conjugation steps), which increases production cost (Krauss et al., 2017; Rehm et al., 2018; Zdarta et al., 2018).

Recently developed in vivo immobilization strategies offer an exciting new concept for enzyme immobilization that holds the promise for cost-effective production of improved industrial biocatalysts (Rehm et al., 2018). Recent progress in understanding the underlying self-assembly mechanism of a diverse range of naturally occurring supramolecular nanostructures has led to the possibility of constructing taskspecific designer scaffolding platforms in vivo. Industrially relevant enzymes of interest can be covalently displayed on the surface and/or incorporated easily within a variety of bio-nanostructures in vivo by genetic engineering of the selfassembling subunits (Rehm et al., 2016; Wilkerson et al., 2018; Schmid-Dannert and López-Gallego, 2019). In contrast to the in vitro methods, the in vivo approaches can display enzymes in a homogeneous and oriented manner on solid supports. These in vivo approaches enable to bypass the harsh and time-consuming immobilization procedures that are often encountered in the in vitro methods. The in vivo formation of solid supports displaying enzymes is implemented intracellularly in bacterial cells by one-step production and, thus, additional cross-linking between the enzymes and solid materials is not needed. This one-pot approach is convenient, efficient, and ultimately enables the low-cost production of robust biocatalysts at a large scale (Rehm et al., 2016).

Several promising biological supramolecular assemblies, such as polyhydroxyalkanoate (PHA) particles (Hooks et al., 2014; Parlane et al., 2016b), virus-like particles (VLPs) (Schwarz et al., 2017; Wilkerson et al., 2018), enzyme-derived nanoparticles (EZPs) (Raeeszadeh-Sarmazdeh et al., 2016; Diaz et al., 2018; Schmid-Dannert and López-Gallego, 2019), membrane vesicles (Rehm et al., 2016; Sharma et al., 2018), and magnetosomes (Jacob and Suthindhiran, 2016; Yan et al., 2017) have been studied to immobilize a variety of functional proteins, including industrially relevant enzymes using recombinant 
fusion technology (Figure 1). Briefly, genetically amenable components of these scaffolds are translationally fused with proteins of interest, such as, e.g., enzymes, and are produced in a range of recombinant expression systems, like various prokaryotic and eukaryotic organisms. These recombinant host cells allow simultaneous protein and scaffold synthesis and subsequent self-assembly of these components. These methods have shown their applicability in the production of immobilized enzymes with improved functionality, presenting a promising means for cost-effective and one-step in vivo enzyme immobilization. Here, we will first review the most promising supramolecular assemblies suitable for in vivo enzyme immobilization and their recent proof-of-concept demonstrations. Then, we will compare the advantages and limitations of PHA particle technology with other biological scaffold-based in vivo enzyme immobilization methods focusing on immobilization of industrially relevant enzymes. Finally, we will discuss innovative methods to expand the utility of the PHA particle technology, including its implementation into continuous-flow catalytic conversions.

\section{UTILIZATION OF VARIOUS SUPRAMOLECULAR ASSEMBLIES AS ENZYME IMMOBILIZATION SUPPORTS}

\section{Polyhydroxyalkanoate}

Polyhydroxyalkanoates are natural biopolyesters, composed of $(R)$-3-hydroxy fatty acids, and are produced by various bacteria in the presence of an excess carbon source, such as glucose (Gonzalez-Miro et al., 2019; Moradali and Rehm, 2020). PHAs are synthesized by PHA synthases and are deposited as spherical polyester inclusions, which serve as an energy and carbon source (Rehm, 2003, 2010). PHA particles vary in size and range between 100 and $500 \mathrm{~nm}$ (Steinmann et al., 2010; Parlane et al., 2016b; Gonzalez-Miro et al., 2019). Poly-(R)-3-hydroxybutyrate (PHB) was the first PHA polymer identified by Lemoigne in 1926 in Bacillus megaterium and is the most common form of PHA

Biological supramolecular assemblies suitable for enzyme immobilization

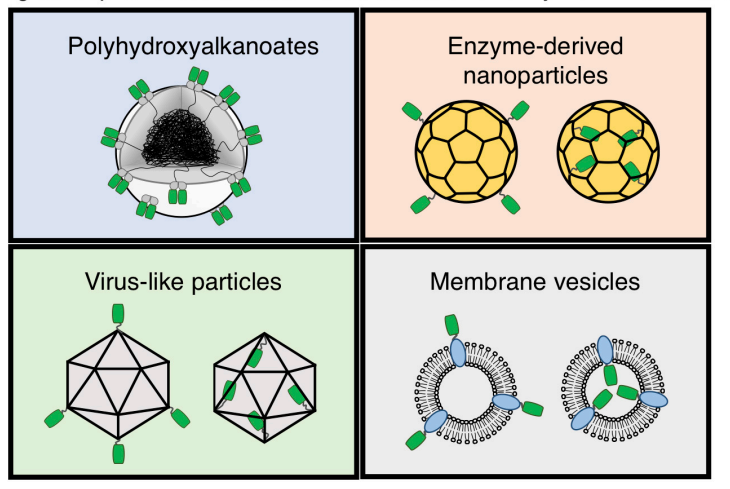

FIGURE 1 | Enzyme (shown in green) immobilization via various biological supramolecular assemblies.
(Kikkawa et al., 2005; Campisano et al., 2008). Generally, each bacterial cell can produce 5-10 PHA particles, the mass of which can contribute up to $90 \%$ of cellular dry weight (Lee, 1996; Koller et al., 2010; Mathuriya and Yakhmi, 2017). The physicochemical properties of PHA particles are significantly influenced by the length and composition of the hydroxyl fatty acids (Mathuriya and Yakhmi, 2017; Gonzalez-Miro et al., 2019). Over 150 different PHA constituents are known (Rehm, 2007; Keshavarz and Roy, 2010; Gonzalez-Miro et al., 2019). The PHAs are classified into three main classes, dependent on the chemical structure and the chain length of the fatty acid monomers: short-chain length PHAs (three to five carbon atoms); medium-chain length PHAs (6-14 carbon atoms); and long-chain length PHAs (>14 carbon atoms) (Mathuriya and Yakhmi, 2017; Ross et al., 2017). Short-chain length PHAs generally have a high level of crystallinity and, thus, are hard and brittle. Medium-chain length PHAs usually have a low melting temperature and crystallinity and, therefore, they are more elastomeric (Philip et al., 2007; Parlane et al., 2016b; Mathuriya and Yakhmi, 2017).

Polyhydroxyalkanoate particles are comprised of an amorphous hydrophobic PHA core surrounded by PHAassociated proteins (PAPs), including PHA synthase (PhaC), phasins (e.g., PhaP and PhaF), structural proteins, PHA depolymerase, structural proteins, and other regulatory proteins (Figure 2A) (Parlane et al., 2016b). Numerous metabolic pathways can provide an array of $(R)$-3-hydroxy fatty acids for the production of PHAs with varying structures and properties as reviewed elsewhere (Meng et al., 2014). PhaC dimers can polymerize these monomer precursors to PHA chains while $\mathrm{PhaC}$ itself remains attached to nascent PHA chains via a covalent thioester bond involving the active site cysteine residue of the PhaC (Peoples and Sinskey, 1989a,b). The covalent link between these two components, namely, the growing hydrophobic PHA chains and the soluble PhaC, eventually leads to amphipathic molecules self-assembling into the spherical PHA particles as shown in scanning electron microscopy (SEM) and transmission electron microscopy (TEM) micrographs (Figure 2B) (Taguchi and Doi, 2004; Rehm, 2007). Another interesting class of PAPs, the phasins, are a type of amphipathic proteins that have several roles in controlling the structure and surface properties of PHA particles (Pieper-Fürst et al., 1994; Wieczorek et al., 1995). Notably, phasins have a high binding affinity to the outer surface of PHA particles in vivo and in vitro mediated by physical adsorption (Tarazona et al., 2019).

The PHA-anchoring characteristics of these PAPs via both covalent interactions (PhaC) and physical adsorption (PhaF and $\mathrm{PhaP}$ ) to the surface of PHA particles have been exploited to fabricate task-specific designer PHA particles using recombinant DNA technology (Hay et al., 2015; Hooks and Rehm, 2015; Jahns and Rehm, 2015; Bello-Gil et al., 2018a,b). PAPs can be translationally fused to target proteins, including industrially relevant enzymes, to enable the recombinant production of functionalized PHA particles in vivo (Figures 2C,D). This approach allows the cost-effective oriented display of immobilized enzymes on the polymeric particulate carrier in one step, ultimately avoiding the laborious chemical cross-linking 
A

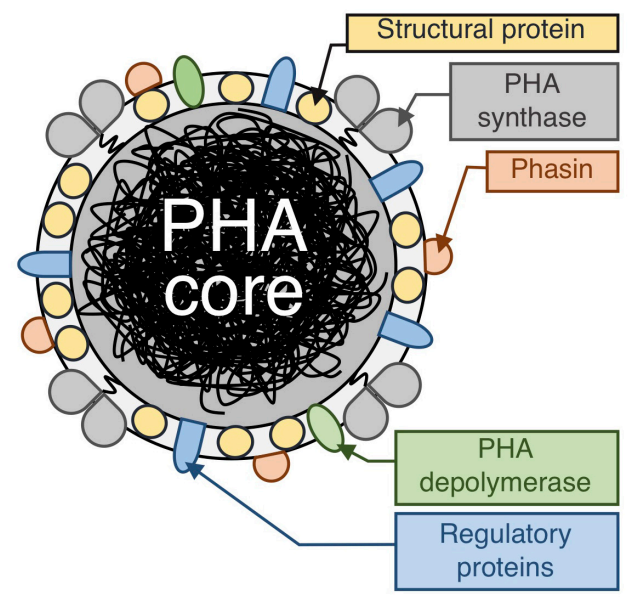

C

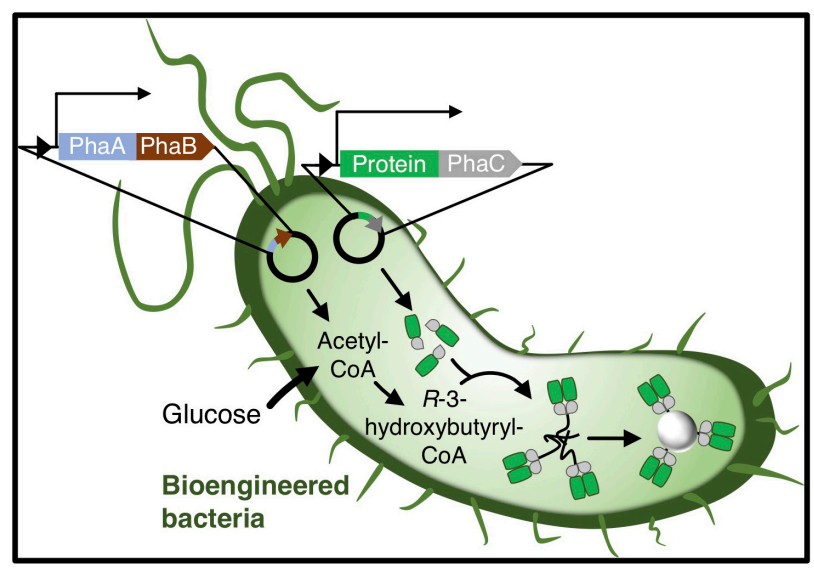

B

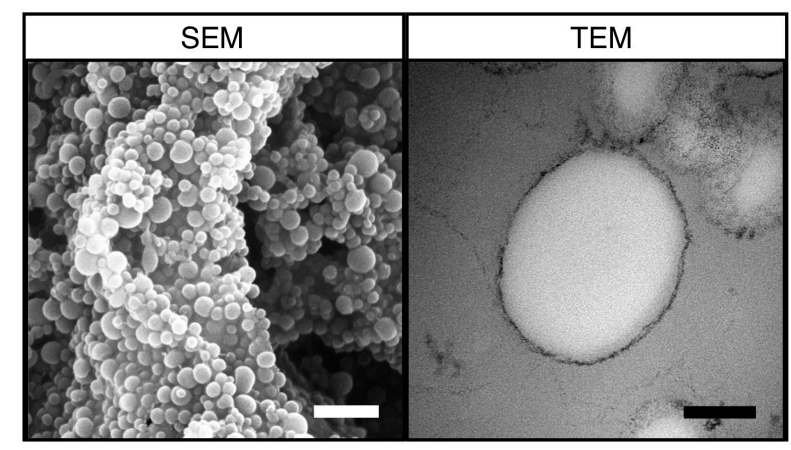

D

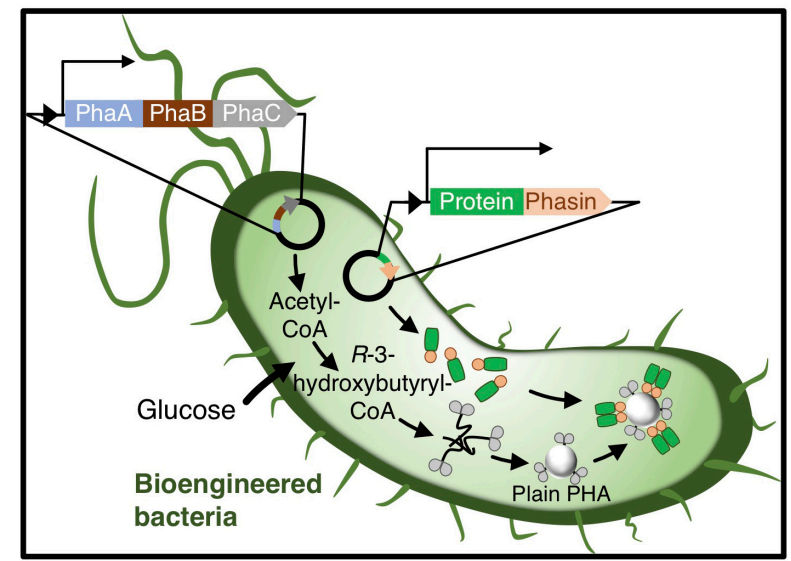

FIGURE 2 | (A) Schematic of a wild-type PHA particle coated by PAPs. (B) SEM and TEM micrographs of PHA particles. White scale bar, 1 $\mu$ m; black scale bar, $100 \mathrm{~nm}$. (C) Self-assembly and functionalization of PHA particles using a PhaC-based gene fusion approach. (D) Self-assembly and functionalization of PHA particles using a phasin-based translational fusion approach.

between enzymes and particles in vitro after isolation (Grage et al., 2009; Hooks et al., 2014; Parlane et al., 2016b).

\section{Virus-Like Particles (VLPs)}

Virus-like particles consist of the viral capsid proteins (CPs; Charlton Hume et al., 2019). The formation of VLPs is a selfassembling process of the viral capsid, which potentially mimics the general structure of the parental virus. However, VLPs do not contain nucleic acids, and, thus, there is no risk of causing infection (Charlton Hume et al., 2019; Janitzek et al., 2019). The CP subunits can be genetically modified for bioconjugation, enabling molecules of interest to be densely displayed or encapsulated in homogeneous spatial orientation (Tan and Jiang, 2017; Charlton Hume et al., 2019). VLPs have made significant advances in various fields, from vaccinology to industrial uses due to their promising characteristics, including monodispersed particle size distribution, defined geometric surfaces, biosafety, and functional programmability (Mohsen et al., 2017; Janitzek et al., 2019). Additionally, the viral capsids are stable over a wide range of environmental conditions, such as temperatures and pHs, which make them suitable for different applications, including industrial biocatalysis (Stanley, 2014; Koch et al., 2015; Brasch et al., 2017; Torbensen et al., 2019). Nevertheless, VLPs are challenging to be abundantly produced at the industrial scale (Mohsen et al., 2017; Tan and Jiang, 2017). A significant drawback of the VLP platform is that the size of the protein attached to, or accommodated within, the particles is limited. This disadvantage precludes the presentation of large functional moieties (Charlton Hume et al., 2019).

\section{Enzyme-Derived Nanoparticles (EZPs)}

Enzyme-derived nanoparticles are highly organized cage-like nanostructures that can be found in both prokaryotic and eukaryotic cells. These naturally evolved protein assemblies can often comprise biomacromolecules such as, e.g., enzymes or inorganic moieties (e.g., iron) that are involved in a range of metabolic and biochemical pathways [peroxidase catalyzed processes (encapsulin), production of vitamin B2 (lumazine synthase), and iron homeostasis (ferritin)] (Arosio et al., 2009; Giessen and Silver, 2017; Azuma et al., 2018). These spherical 
nanostructures are highly attractive owing to their particle uniformity, biocompatibility, and precise controllability. Being able to fine-tune the morphological architecture and functions of these particulate scaffolds has made them excellent candidates for the design of biocatalytic nanoreactors (Raeeszadeh-Sarmazdeh et al., 2016; Lee, 2018; Schmid-Dannert and López-Gallego, 2019). EZPs can be reprogrammed to incorporate various foreign biological functions such as, e.g., enzymes of industrial interest. Both chemical and bioengineering methods can be utilized to modify the scaffold protein (SP) subunits of EZPs to enable spatial organization of enzymes within and/or on the surface of the EZPs. This design space enables the fabrication of various artificial multienzyme complexes for industrial uses (SchmidDannert and López-Gallego, 2019). Although these scaffolds have been manufactured in numerous recombinant expression systems (e.g., various prokaryotic and eukaryotic organisms), they have been mainly assembled in Escherichia coli strains (Diaz et al., 2018). Advances in protein engineering in recent years harnessed the unique structural assembly of EZPs to enable the de novo and in silico design of novel EZPs (King et al., 2014; Bale et al., 2016; Hsia et al., 2016; Jakobson et al., 2017; Xu et al., 2019).

\section{Extracellular Membrane Vesicles (EMVs)}

Extracellular membrane vesicles are lipid membrane-derived compartments and are found in all domains of life (Raposo and Stoorvogel, 2013; Gill et al., 2018; Lee, 2019). Their sizes are in the range of $20-1000 \mathrm{~nm}$ in diameter (van der Pol et al., 2012; Gill et al., 2018), and they mainly serve as carrier vehicles to mediate cell-to-cell communication by transporting biological cargo as, for example, DNA, RNA, and proteins (Burkova et al., 2018; Longatti et al., 2018). The classification of these functionally and structurally diverse EMVs, including the bacterial outer membrane vesicles, microvesicles, and exosomes, has been thoroughly reviewed (van der Pol et al., 2012; Cocucci and Meldolesi, 2015; Gill et al., 2018; Greening and Simpson, 2018). Although the exact underlying mechanism on how different EMVs are formed is still unknown, recent studies show that various recombinantly modified protein production cell lines, including well-established E. coli production strains, can produce task-specific EMVs. It was shown that foreign proteins of interest, such as enzymes, could be incorporated into the outer surface or within the inner surface of the EMVs via membraneanchoring motifs, such as transmembrane domains, using genetic engineering to create respective translational fusions (Alves et al., 2015; Su et al., 2017). This approach led to numerous pharmaceutical and bioremediation applications (Ohno et al., 2013; Alves et al., 2016, 2018; Arrighetti et al., 2019). In addition, EMVs are relatively stable in ambient environments and can be manufactured cost-effectively (Collins, 2011). However, isolation and purification of EMVs still require expensive and laborious ultracentrifugation steps, which potentially impact the structural integrity of EMVs and which prohibit industrial scale production (Su et al., 2017; Longatti et al., 2018; Bruce et al., 2019).

\section{Magnetosomes}

Bacterial magnetosomes are inclusions (20-60 nm) present in magnetotactic bacteria comprised of magnetic mineral crystals (iron oxide or iron sulfide nanoparticle) surrounded by a phospholipid double-layered membrane (Dieudonné et al., 2019). The magnetosome membrane is derived from the cytoplasmic membrane and can protect the iron crystal from oxidation (Dieudonné et al., 2019). Many magnetosome membrane proteins (e.g., MamB, MamM, MamH, and MamZ) are involved in magnetosome formation and dictate the iron uptake into the vesicle (Lohße et al., 2014; Uebe and Schüler, 2016; Dieudonné et al., 2019). Meanwhile, the size and morphology of the magnetosomes are controlled by another set of magnetosome membrane proteins as, for example, MamC/Mms13, MamD, MamF, MamG, MamR, MamS, Mms6, and MmsF (Uebe and Schüler, 2016; Islam et al., 2018; Dieudonné et al., 2019). Interestingly, magnetosomes can be functionalized in vivo by fusing foreign proteins of interest to the magnetosome membrane proteins, such as MamC/Mms13, MagA, and Mms16 (Arakaki et al., 2008; Ren et al., 2018). The translational fusion of functional proteins to these transmembrane proteins of magnetosomes has led to numerous successful prototypes in a wide range of applications, including of industrial uses (Ginet et al., 2011; Sugamata et al., 2013; Honda et al., 2015a,b; Xiang et al., 2017; Mickoleit and Schüler, 2018). The inherent magnetic characteristics of magnetosomes make them very useful in some situations, especially for implementation in magneticfield-related technologies, such as magneto-immunoassays and biomedical imaging (Ren et al., 2018). The implementation of magnetosomes also allows rapid magnetic separation of the functionalized particulate scaffolds from the bulk fluids (Rehm et al., 2018). However, several technical issues encountered in manipulating and cultivating magnetosomes represent some of the main hurdles in expanding the use of this exciting technology (Bakhshi et al., 2016).

\section{BIOLOGICAL SUPRAMOLECULAR ASSEMBLIES AS BIOCATALYST SUPPORTS}

Table 1 summarizes recent studies describing in vivo immobilization approaches for a range of industrially relevant enzymes, detailing their functional performance and robustness in various experimented conditions.

\section{COMPARATIVE ANALYSIS OF IN VIVO IMMOBILIZATION STRATEGIES}

\section{Advantages and Current Limitations of the Recombinant PHA Particle Technology}

Genetic engineering of PAPs represents an interesting approach for enzyme immobilization on PHA particles. Foreign proteins of interest can be translationally fused to the $\mathrm{N}$ - or C-terminus, or both termini of PAPs. The broad applicability and versatility of this approach also allows for the attachment of more than one enzyme to the PHA particle surface (Chen S. et al., 2014; 
TABLE 1 | Biological supramolecular assemblies engineered for in vivo immobilization of industrially relevant enzymes.

\begin{tabular}{|c|c|c|c|c|}
\hline $\begin{array}{l}\text { Type of biological } \\
\text { scaffolds and their } \\
\text { anchoring motifs }\end{array}$ & $\begin{array}{l}\text { Target enzyme } \\
\text { (Origin) } \\
\text { (Gene fusion site) } \\
\text { (Production host) }\end{array}$ & Catalytic performance & Stability & References \\
\hline \multicolumn{5}{|c|}{ Polyhydroxyalkanoates (PHAs) } \\
\hline \multirow[t]{5}{*}{$\begin{array}{l}\text { PHAs via Cupriavidus } \\
\text { necator PHA synthase } \\
\text { PhaC }\end{array}$} & $\begin{array}{l}\text { a-Amylase } \\
\text { (Bacillus licheniformis) } \\
\text { (C-terminus) } \\
\text { [E. coli Origami B (DE3)] }\end{array}$ & $\begin{array}{l}\text { - Consistent with the reported activity of soluble counterpart. } \\
\text { - Michaelis-Menten constant }\left(K_{m}\right) \text { of immobilized } \alpha \text {-amylase catalyzing starch } \\
\text { degradation: } 5 \mu \mathrm{M} \text {. } \\
\text { - } K_{m} \text { of soluble } \alpha \text {-amylase reported in the literature catalyzing starch degradation: } \\
9.6 \mu \mathrm{M} \text {. } \\
\text { - Specific activity of immobilized } \alpha \text {-amylase catalyzing starch degradation: } 506 \mathrm{mU} / \mathrm{mg} \text { of } \\
\text { fusion protein. }\end{array}$ & $\begin{array}{l}\text { - Tolerant to extreme pH } \\
\text { and temperature } \\
\text { conditions. }\end{array}$ & Rasiah and Rehm, 2009 \\
\hline & $\begin{array}{l}\text { Hexavalent chromium } \\
\text { reductase, NemA } \\
\text { (E. coli) } \\
\text { (N-terminus) } \\
{[\text { E. coli BL21(DE3)] }}\end{array}$ & $\begin{array}{l}\text { - Showed activity to their substrate but at varying efficiencies. } \\
K_{m} \text { of immobilized NemA for the reduction of Cr(VI): } 94 \pm 26 \mu \mathrm{M} \text {. } \\
\text { - } K_{m} \text { of soluble NemA for the reduction of } \mathrm{Cr}(\mathrm{VII}): 16 \pm 8.6 \mu \mathrm{M} \text {. } \\
\text { - } K_{m} \text { of immobilized NemA for the reduction of } \mathrm{NADH}: 490 \pm 30 \mu \mathrm{M} \text {. } \\
\text { - } K_{m} \text { of soluble NemA for the reduction of } \mathrm{NADH}: 450 \pm 30 \mu \mathrm{M}\end{array}$ & $\begin{array}{l}\text { - No observable reduction } \\
\text { in activity after } 36 \text { weeks } \\
\text { of storage at } 4^{\circ} \mathrm{C} \text {. }\end{array}$ & Robins et al., 2013^ \\
\hline & $\begin{array}{l}\text { N-acetylglucosamine } \\
\text { 2-epimerase, } \\
\text { SIr1975 } \\
\text { (Synechocystis sp. } \\
\text { PCC 6803) } \\
\text { (N-terminus) } \\
\text { [E. coli BL21(DE3)] } \\
\text { N-acetylneuraminic } \\
\text { acid aldolase, NanA } \\
\text { (E. coli) } \\
\text { (C-terminus) } \\
\text { [E. coli BL21(DE3)] }\end{array}$ & $\begin{array}{l}\text { - Artificial enzyme cascading system had overall conversion yield of } \sim 22 \% \text {, compared to } \\
\text { that of traditional method at } \sim 33 \% \text { catalyzing } N \text {-acetyl-D-glucosamine conversion to } \\
N \text {-acetylneuraminic acid. } \\
\text { - Specific activity of immobilized SIr1975 catalyzing } N \text {-acetyl-D-glucosamine conversion } \\
\text { to } N \text {-acetyl-D-mannosamine: } 1.76 \pm 0.38 \mathrm{U} / \mathrm{mg} \text { fusion protein. } \\
\text { - Specific activity of immobilized SIr1975 catalyzing } N \text {-acetyl-D-glucosamine conversion } \\
\text { to } N \text {-acetyl-D-mannosamine when co-immobilized with NanA: } 0.58 \pm 0.07 \mathrm{U} / \mathrm{mg} \text { of } \\
\text { fusion protein. } \\
\text { - Specific activity of immobilized NanA catalyzing } N \text {-acetyl-D-mannosamine conversion } \\
\text { to } N \text {-acetylneuraminic acid: } 42.6 \pm 6.9 \mathrm{U} / \mathrm{mg} \text { of fusion protein. } \\
\text { - Specific activity of immobilized NanA catalyzing } N \text {-acetyl-D-mannosamine conversion } \\
\text { to } N \text {-acetylneuraminic acid when co-immobilized with SIr1975: } 81.9 \pm 19 \mathrm{U} / \mathrm{mg} \text { of } \\
\text { fusion protein. }\end{array}$ & $\begin{array}{l}\text { - Retained } \sim 80 \% \text { of its } \\
\text { initial activity after five } \\
\text { reaction cycles. }\end{array}$ & Hooks et al., $2013^{*}$ \\
\hline & $\begin{array}{l}\text { Lipase B } \\
\text { (Candida antarctica) } \\
\text { (N-terminus) } \\
\text { [E. coli BL21 } \\
\text { (DE3)] }\end{array}$ & $\begin{array}{l}\text { - Retained but exhibited lower activity ( } 30-40 \%) \text { catalyzing glycerol tributyrate } \\
\text { hydrolysis when compared to the commercially available immobilized lipase (Novozyme } \\
\text { 435). }\end{array}$ & $\begin{array}{l}\text { - Retained initial activity } \\
\text { after } 7 \text { weeks of storage } \\
\text { at } 4^{\circ} \mathrm{C} \text {. }\end{array}$ & Jahns and Rehm, 2015^^ \\
\hline & $\begin{array}{l}\text { Carbonic anhydrase } \\
\text { (Desulfovibrio vulgaris } \\
\text { str. "Miyazaki F"), DvCA } \\
\text { (C-terminus) } \\
\text { [E. coli BL21(DE3)] }\end{array}$ & $\begin{array}{l}\text { - Retained but exhibited lower activity when compared to the commercially available } \\
\text { soluble counterpart. } \\
\text { - Specific activity of immobilized DvCA catalyzing the hydration of carbon dioxide: } \\
114 \mathrm{U} / \mathrm{mg} \text { of enzyme (highest at } 211 \mathrm{U} / \mathrm{mg} \text { of enzyme). }\end{array}$ & $\begin{array}{l}\text { - Tolerant to alkaline and } \\
\text { elevated temperature } \\
\text { environments. }\end{array}$ & Hooks and Rehm, 2015* \\
\hline
\end{tabular}


TABLE 1 | Continued

\begin{tabular}{|c|c|c|c|c|}
\hline \multirow[t]{5}{*}{$\begin{array}{l}\text { Type of biological } \\
\text { scaffolds and their } \\
\text { anchoring motifs }\end{array}$} & $\begin{array}{l}\text { Target enzyme } \\
\text { (Origin) } \\
\text { (Gene fusion site) } \\
\text { (Production host) }\end{array}$ & Catalytic performance & Stability & References \\
\hline & $\begin{array}{l}\text { Lipase M37 } \\
\text { (Photobacterium } \\
\text { lipolyticum) } \\
\text { (C-terminus) } \\
\text { (E. coli XL1-Blue) }\end{array}$ & $\begin{array}{l}\text { - Consistent with the reported activity of soluble counterpart but exhibited narrow substrate chain } \\
\text { length specificity. } \\
\text { - Specific activity of immobilized lipase M37 catalyzing p-nitrophenyl esters conversion to } \\
p \text {-nitrophenol: } 108.4 \pm 2.5 \mathrm{U} / \mathrm{g} \text { of dry weight PHA particles. }\end{array}$ & $\begin{array}{l}\text { - Enhanced thermal } \\
\text { stability and retained } \\
\text { initial activity after } \\
4 \text { weeks of storage at } \\
4^{\circ} \mathrm{C} \text {. }\end{array}$ & Yang et al., $2015^{\star}$ \\
\hline & $\begin{array}{l}\text { Alkaline } \\
\text { polygalacturonate } \\
\text { lyase, PGL } \\
\text { (Bacillus subtilis) } \\
\text { (C-terminus) } \\
\text { [E. coli BL21(DE3)] }\end{array}$ & $\begin{array}{l}\text { - Retained } 85 \% \text { of the catalytic activity of soluble counterpart. } \\
\text { - Specific activity of immobilized PGL catalyzing polygalacturonic acid conversion to unsaturated } \\
\text { oligo-galacturonic acid: } 184.67 \pm 11.53 \mathrm{U} / \mathrm{mg} \text { of enzyme. } \\
\text { - Specific activity of soluble PGL catalyzing polygalacturonic acid conversion to unsaturated } \\
\text { oligo-galacturonic acid: } 215.93 \pm 8.95 \mathrm{U} / \mathrm{mg} \text { of enzyme. }\end{array}$ & $\begin{array}{l}\text { - Retained } \sim 60 \% \text { of its } \\
\text { initial activity after eight } \\
\text { reaction cycles. } \\
\text { - Moderately enhanced } \\
\text { thermal and pH stability. }\end{array}$ & Ran et al., $2017^{\star}$ \\
\hline & $\begin{array}{l}\text { Tyrosinase } \\
\text { (Verrucomicrobium } \\
\text { spinosum) } \\
\text { (C-terminus) } \\
\text { [E. coli BL21(DE3)] }\end{array}$ & $\begin{array}{l}\text { - Immobilized tyrosinase showed enhanced specific activity catalyzing L-tyrosine conversion to } \\
\text { L-dopaquinone when compared to its soluble counterpart. } \\
\text { - Monophenolase activity of immobilized tyrosinase catalyzing L-tyrosine conversion to } \\
\text { 3,4-dihydroxyphenyl-L-alanine: } 9155.88 \pm 312.57 \mathrm{U} / \mathrm{g} \text { of enzyme. } \\
\text { - Monophenolase activity of soluble tyrosinase catalyzing L-tyrosine conversion to } \\
\text { 3,4-dihydroxyphenyl-L-alanine: } 2185.50 \pm 74.61 \mathrm{U} / \mathrm{g} \text { of enzyme. } \\
\text { - Diphenolase activity of immobilized tyrosinase catalyzing 3,4-dihydroxyphenyl-L-alanine conversion } \\
\text { to L-dopaquinone: } 297.27 \pm 21.25 \mathrm{U} / \mathrm{g} \text { of enzyme. } \\
\text { - Diphenolase activity of soluble tyrosinase catalyzing 3,4-dihydroxyphenyl-L-alanine conversion to } \\
\text { L-dopaquinone: } 32.10 \pm 3.10 \mathrm{U} / \mathrm{g} \text { of enzyme. }\end{array}$ & $\begin{array}{l}\text { - Retained its initial } \\
\text { activity up to six } \\
\text { reaction cycles. } \\
\text { - Widened optimal } \\
\text { operating temperature } \\
\text { range. }\end{array}$ & Tan et al., 2019* \\
\hline & $\begin{array}{l}\text { D-tagatose-3- } \\
\text { epimerase, DTE } \\
\text { (Pseudomonas cichorii) } \\
\text { (C-terminus) } \\
\text { [E. coli ClearColi BL21 } \\
\text { (DE3)] }\end{array}$ & $\begin{array}{l}\text { - Had overall conversion yield of } \sim 33 \% \text { catalyzing } D \text {-fructose conversion to D-allulose. } \\
\text { - Specific activity of immobilized DTE catalyzing D-fructose conversion to D-allulose: } \\
357.77 \pm 16.66 \mathrm{U} / \mathrm{mg} \text { of enzyme. } \\
\text { - Specific activity of soluble DTE catalyzing D-fructose conversion to D-allulose: } \\
531.29 \pm 31.87 \mathrm{U} / \mathrm{mg} \text { of enzyme. }\end{array}$ & $\begin{array}{l}\text { - Retained } ~ 80 \% \text { of its } \\
\text { initial activity after eight } \\
\text { reaction cycles. } \\
\text { - Exhibited similar } \\
\text { thermal and pH stability } \\
\text { when compared to its } \\
\text { soluble counterpart. }\end{array}$ & Ran et al., 2019* \\
\hline \multirow[t]{2}{*}{$\begin{array}{l}\text { PHAs via } \\
\text { Pseudomonas putida } \\
\text { phasin PhaF }\end{array}$} & $\begin{array}{l}\beta \text {-Galactosidase, } \beta \text {-gal } \\
\text { (E. coli) } \\
\text { (N-terminus) } \\
\text { (Pseudomonas putida } \\
\text { GPG-Tc6) }\end{array}$ & $\begin{array}{l}\text { - Showed specific activity to its substrate. } \\
\text { - Specific activity of immobilized } \beta \text {-gal catalyzing the hydrolysis of } \\
\text { o-nitro-phenyl- } \beta \text {-D-galactopyranoside: } 2.8 \times 10^{5} \mathrm{U} / \mathrm{mg} \text { of enzyme. } \\
\text { - Specific activity of soluble } \beta \text {-gal catalyzing the hydrolysis of } 0 \text {-nitro-phenyl- } \beta \text {-D-galactopyranoside } \\
\text { cleaved from } \beta \text {-gal displaying PHA particles: } 2.2 \times 10^{5} \mathrm{U} / \mathrm{mg} \text { of enzyme. }\end{array}$ & $\bullet N / A$ & Moldes et al., 2004* \\
\hline & $\begin{array}{l}\text { Cry1Ab toxin } \\
\text { (Bacillus thuringiensis) } \\
\text { (N-terminus) } \\
\text { (Pseudomonas putida } \\
\text { GPG-Tc6) }\end{array}$ & $\begin{array}{l}\text { - Immobilized Cry1Ab showed 7.2-fold less insecticidal activity against the larvae of Sesamia } \\
\text { nonagrioides when compared with its soluble counterpart. }\end{array}$ & - N/A & Moldes et al., $2006^{\wedge *}$ \\
\hline
\end{tabular}


TABLE 1 | Continued

\section{Type of biological} scaffolds and their anchoring motifs

\section{Target enzyme}

(Origin)

(Gene fusion site)

(Production host)

PHAs via Cupriavidus D-hydantoinase,

necator phasin PhaP

D-HDT

(Agrobacterium

radiobacter NRRL

B11291)

(N-terminus)

(E. coli DH5a)

Lysine decarboxylase,

CadA

(E. coli)

(N-terminus)

[E. coli BL21(DE3)]

PHAs via Cupriavidus necator PHA synthase PhaC and

PHAs via Cupriavidus

Organophosphorus hydrolase, OpdA (Pseudoalteromonas necator phasin PhaP

N-terminus)

[E. coli BL21(DE3)]

\section{Virus-like particles (VLPs)}

Bacteriophage MS2 CP Pyridoxal

subunit

phosphate-dependent

tryptophanase, TnaA

(E. coli)

(N- and C-termini)

[E. coli BL21(DE3) Star]

\section{Catalytic performance}

Stability

References

- Immobilized D-HDT showed similar specific activity in catalyzing D,L-hydroxyphenyl hydantoin

conversion to $N$-carbamoyl-L-p-hydroxy phenylglycine with its soluble counterpart.

- Ranged between 80 and $107 \mathrm{U}$ due to varying biosynthesis conditions of in vivo functionalized

PHA particles.

- Consistent with its soluble counterpart.

- Specific activity of immobilized CadA catalyzing lysine conversion to cadaverine:

$179.5 \pm 1.8 \mathrm{U} / \mathrm{mg}$ of enzyme.

- Specific activity of soluble CadA catalyzing lysine conversion to cadaverine: $95.15 \pm 9.5 \mathrm{U} / \mathrm{mg}$ of enzyme.

- Km of OpdA immobilized using PhaC catalyzing paraoxon hydrolysis: $6.188 \pm 2.490 \mathrm{mM}$.

- $K_{m}$ of OpdA immobilized using PhaP catalyzing paraoxon hydrolysis: $6.116 \pm 1.299 \mathrm{mM}$.

- $K_{m}$ of soluble OpdA catalyzing paraoxon hydrolysis: $3.203 \pm 0.929 \mathrm{mM}$.

- $k_{\text {cat }}$ of OpdA immobilized using PhaC catalyzing paraoxon hydrolysis: $11.904 \pm 3.893 \mathrm{~s}^{-1}$

- $k_{\text {cat }}$ of OpdA immobilized using PhaP catalyzing paraoxon hydrolysis: $11.223 \pm 1.752 \mathrm{~s}^{-1}$.

- $k_{\text {cat }}$ of soluble OpdA catalyzing paraoxon hydrolysis: $3.0 \pm 0.526 \mathrm{~s}^{-1}$

- $k_{\text {cat }} / K_{m}$ of OpdA immobilized using PhaC catalyzing paraoxon hydrolysis: $1961 \pm 138 \mathrm{M}^{-1} \mathrm{~s}^{-1}$

- $K_{\text {cat }} / K_{m}$ of OpdA immobilized using PhaP catalyzing paraoxon hydrolysis: $1850 \pm 104 \mathrm{M}^{-1} \mathrm{~s}^{-1}$.

- $K_{\text {cat }} / K_{m}$ of soluble OpdA catalyzing paraoxon hydrolysis: $935 \pm 89 \mathrm{M}^{-1} \mathrm{~s}^{-1}$

- Specific activity of OpdA immobilized using PhaC catalyzing paraoxon hydrolysis:

$0.096 \pm 0.0047 \mathrm{U} / \mathrm{mg}$ of enzyme.

- Specific activity of OpdA immobilized using PhaP catalyzing paraoxon hydrolysis:

$0.109 \pm 0.0014 \mathrm{U} / \mathrm{mg}$ of enzyme.

- Specific activity of OpdA immobilized using PhaC and PhaP catalyzing paraoxon hydrolysis:

$0.112 \pm 0.0044 \mathrm{U} / \mathrm{mg}$ of enzyme.

- Specific activity of soluble OpdA catalyzing paraoxon hydrolysis: $1.648 \pm 0.222 \mathrm{U} / \mathrm{mg}$ of

enzyme.

- Artificial enzyme cascading system comprised of covalently immobilized TnaA and FMO

showed enhanced overall conversion yield catalyzing L-tryptophan conversion to indigo when

compared to the soluble controls.

Moderately enhanced
Retained $\sim 95 \%$ of its initial activity after 1 week of

storage at $25^{\circ} \mathrm{C}$, compared

to its soluble counterpart

$(\sim 5 \%)$.

Chen S. Y. et at, 2014*

cycles. Enhanced stability

Retained its initial activity

up to five reaction cycles.

- Enhanced stability

particularly under acidic

conditions.

ctivity after 10 repeated

use cycles.

Sen and Silver, 2016^^ 
Type of biological Target enzyme

Catalytic performance

Stability

References

\section{their anchoring (Gene fusion site)}

motifs

(Production host)

Flavin-mononucleotide and

nicotinamide adenine dinucleotide

phosphate dependent containing

monooxygenase, FMO

(Methylophaga sp. Strain SK1)

(N- and C-termini)

[E. coli BL21(DE3) Star]

Bacteriophage P22 Alcohol dehydrogenase D

CP subunit (Pyrococcus furiosus)

(C-terminus)

[E. coli BL21(DE3)]

Hydrogenase 1 subunit $A$ and subunit $B$, HyaA and $\mathrm{HyaB}$

(E. coli)

(C-terminus)

[E. coli BL21(DE3)]

Parvovirus B19 CP Lipase, Bp1A

subunit (Bacillus pumilus)

( $\mathrm{N}$ - and C-termini)

[E. coli BL21(DE3)]

- Showed specific activity for the reduction of 3-hydroxy-2-butanone to 2,3-butanediol.

80-270-fold higher than the reported activity of soluble counterpart for hydrogen production. - Catalytic activity of immobilized hydrogenase for hydrogen production: $3218 \pm 394 \mathrm{nmol} \mathrm{H} / 2 \mathrm{mg}$ min.

- Catalytic activity of the soluble hydrogenase for hydrogen production reported in the literature: 12-38 nmol $\mathrm{H}_{2} / \mathrm{mg}$ min.

- Showed specific activity catalyzing the hydrolysis of 4-nitrophenyl acetate but lower when compared to its soluble counterpart.

- Specific activity of immobilized Bp1A catalyzing the hydrolysis of 4-nitrophenyl acetate:

$9.5 \pm 1.4 \mathrm{U} / \mu \mathrm{mol}$ of enzyme.

- Specific activity of soluble Bp1A catalyzing the hydrolysis of 4-nitrophenyl acetate: $202 \pm 0.4 \mathrm{U} / \mu \mathrm{mol}$ enzyme.

$\alpha$-Glucosidase, Ima1p (Saccharomyces cerevisiae) (C-terminus)

[E. coli BL21(DE3)]

$\begin{array}{ll}\text { Cowpea chlorotic } & \text { Lysozyme } \\ \text { mottle virus CP } & \text { (Enterobacteria phage T4) } \\ \text { subunit } & \text { (C-terminus) } \\ & {[\text { E. coli BLR(DE3) pLysS }]}\end{array}$

- Threefold increase in catalytic activity when compared to its soluble counterpart.

- Catalytic activity of immobilized Ima1p catalyzing 4-nitrophenyl- $\alpha$-D-glucopyranoside hydrolysis: $2.1 \pm 0.05 \mathrm{mM} / \mathrm{min} / \mathrm{mg}$

- Catalytic activity of soluble Ima1p catalyzing 4-nitrophenyl- $\alpha$-D-glucopyranoside hydrolysis:

$0.67 \pm 0.02 \mathrm{mM} / \mathrm{min} / \mathrm{mg}$

- $K_{m}$ of immobilized Ima1p catalyzing 4-nitrophenyl- $\alpha$-D-glucopyranoside hydrolysis:

$1.92 \pm 0.13 \mathrm{mM}$.

- $K_{m}$ of soluble Ima1p catalyzing 4-nitrophenyl- $\alpha$-D-glucopyranoside hydrolysis: $1.72 \pm 0.16 \mathrm{mM}$.

- Showed catalytic activity catalyzing the degradation of fluorescently labeled $M$. luteus cell walls

but $\sim 7$-fold less active than its soluble counterpart.

- Catalytic activity of immobilized lysozyme catalyzing the degradation of fluorescently labeled

M. luteus cell walls: $\sim 400$ arbitrary unit (AU)/min

- Catalytic activity of soluble lysozyme catalyzing the degradation of fluorescently labeled M. luteus cell walls: $\sim 2800 \mathrm{AU} / \mathrm{min}$

- No loss in activity at $25^{\circ} \mathrm{C} \quad$ Patterson et al., 2015^ was observed.

- Showed resistance against Jordan et al., 2016* proteolytic and thermal inactivation.

- Enhanced thermal stability. Bustos-Jaimes et al.

- First-order rate constant of $2017^{*}$

degradation of immobilized

lipase at $40^{\circ} \mathrm{C}$ :

$0.68 \pm 0.11 \mathrm{~h}^{-1}$.

- First-order rate constant of degradation of soluble lipase at $40^{\circ} \mathrm{C}$ : $4.82 \pm 0.37 \mathrm{~h}^{-1}$

- Impaired thermal stability. Cayetano-Cruz et al. 2018

Schoonen et al., 2017* - N/A 
TABLE 1 | Continued

\begin{tabular}{lll}
\hline $\begin{array}{l}\text { Type of biological } \\
\text { scaffolds and their }\end{array}$ & $\begin{array}{l}\text { Target enzyme } \\
\text { (Origin) }\end{array}$ & Catalytic performance \\
anchoring motifs & $\begin{array}{l}\text { (Gene fusion site) } \\
\text { (Production host) }\end{array}$ & \\
&
\end{tabular}

\section{Enzyme-derived nanoparticles (EZPs)}

\section{Bacillus \\ Endoglucanase CelA}

stearothermophilus

pyruvate

dehydrogenase

multienzyme complex

E2 core SP subunit

functionalized with

elastin-like peptide

(ELP-E2)

Citrobacter freundii

Pdu bacterial

microcompartment SP

subunit

(D18 or P18)

(Clostridium

thermocellum)

(C-terminus)

[E. coli BL21(DE3)]

(E. coli)

(C-terminus)

[E. coli BL21(DE3)]

Glycerol

(E. coli)

(N-terminus)

[E. coli BL21(DE3)
$\beta$-Galactosidase, $\beta$-gal

dehydrogenase, GldA

pLysS]

Dihydroxyacetone

kinase, DhaK

(E. coli)

(N-terminus)

[E. coli BL21(DE3)

pLysS]

Methylglyoxal synthas

MgsA

(E. coli)

( $\mathrm{N}$-terminus)

[E. coli BL21(DE3)

pLysS]

1,2-propanediol

oxidoreductase, FucO

(E. coli)

(N-terminus)

[E. coli BL21(DE3)

pLysS]
- Immobilized CelA on ELP-E2 nanoparticles increased the amount of reduced sugar compared to its soluble counterpart.

- Catalytic activity of immobilized CelA catalyzing cellulose hydrolysis: $\sim 17 \mu \mathrm{mol} / \mathrm{h}$

- Catalytic activity of soluble CelA catalyzing cellulose hydrolysis: $\sim 14 \mu \mathrm{mol} / \mathrm{h}$.

- Immobilized $\beta$-gal on ELP-E2 nanoparticles showed catalytic activity visualized by the change in the color of substrate into yellow due to the release of $o$-nitrophenol.

Co-immobilization or aggregation of tagged enzymes catalyzing glycerol conversion to 1,2-propanediol resulted in enhanced conversion yield in vivo compared to the soluble counterpart.

- A reduction of $90 \%$ in the specific activity of GldA bearing D18 when compared to the untagged control catalyzing glycerol conversion to dihydroacetone.

- A reduction of 55\% in the specific activity of GldA bearing P18 when compared to the untagged control catalyzing glycerol conversion to dihydroacetone.

- Specific activity of immobilized DhaK bearing D18 catalyzing dihydroacetone conversion to dihydroacetone phosphate: $\sim 5.5 \mu \mathrm{mol} / \mathrm{min} / \mathrm{mg}$.

- Specific activity of immobilized DhaK bearing P18 catalyzing dihydroacetone conversion to dihydroacetone phosphate: $\sim 5.0 \mu \mathrm{mol} / \mathrm{min} / \mathrm{mg}$.

- Specific activity of untagged DhaK catalyzing dihydroacetone conversion to dihydroacetone phosphate: $\sim 5.1 \mu \mathrm{mol} / \mathrm{min} / \mathrm{mg}$.

- Specific activity of immobilized MgsA bearing D18 catalyzing dihydroacetone phosphate conversion to methylglyoxal: $\sim 14 \mu \mathrm{mol} / \mathrm{min} / \mathrm{mg}$.

- Specific activity of immobilized MgsA bearing P18 catalyzing dihydroacetone phosphate conversion to methylglyoxal: $\sim 13 \mu \mathrm{mol} / \mathrm{min} / \mathrm{mg}$

- Specific activity of untagged Mgs catalyzing dihydroacetone phosphate conversion to methylglyoxal: $\sim 16 \mu \mathrm{mol} / \mathrm{min} / \mathrm{mg}$.

- Specific activity of immobilized GldA bearing D18 catalyzing methylglyoxal conversion to lactaldehyde: $\sim 0.4 \mu \mathrm{mol} / \mathrm{min} / \mathrm{mg}$

- Specific activity of immobilized GldA bearing P1 catalyzing methylglyoxal conversion to lactaldehyde: $\sim 0.9 \mu \mathrm{mol} / \mathrm{min} / \mathrm{mg}$

- Specific activity of untagged GldA catalyzing methylglyoxal conversion to lactaldehyde: $\sim 2.1 \mu \mathrm{mol} / \mathrm{min} / \mathrm{mg}$

- Specific activity of immobilized FucO bearing D18 catalyzing lactaldehyde conversion to 1,2-propanediol: $\sim 6 \mu \mathrm{mol} / \mathrm{min} / \mathrm{mg}$

- Specific activity of immobilized FucO bearing P18 catalyzing lactaldehyde conversion to 1,2-propanediol:

$\sim 2.5 \mu \mathrm{mol} / \mathrm{min} / \mathrm{mg}$.

- Specific activity of untagged FucO catalyzing lactaldehyde conversion to 1,2-propanediol: $\sim 10.0 \mu \mathrm{mol} / \mathrm{min} / \mathrm{mg}$
Immobilized CelA on ELP-E2 nanoparticles remained functional up to $70^{\circ} \mathrm{C}$.

Chen et al., $2015^{\star}$

- N/A

Lee et al., 2016* 
TABLE 1 | Continued

\begin{tabular}{|c|c|c|c|c|}
\hline $\begin{array}{l}\text { Type of biological } \\
\text { scaffolds and their } \\
\text { anchoring motifs }\end{array}$ & $\begin{array}{l}\text { Target enzyme } \\
\text { (Origin) } \\
\text { (Gene fusion site) } \\
\text { (Production host) }\end{array}$ & Catalytic performance & Stability & References \\
\hline $\begin{array}{l}\text { Salmonella enterica } \\
\text { Pdu bacterial } \\
\text { microcompartment SP } \\
\text { subunit }\end{array}$ & $\begin{array}{l}\beta \text {-Galactosidase, } \beta \text {-gal } \\
\text { (E. coli) } \\
\text { (N-terminus) } \\
\text { (Salmonella enterica) } \\
\text { Glycerol } \\
\text { dehydrogenase, GldA } \\
\text { (E. coli) } \\
\text { (N-terminus) } \\
\text { (Salmonella enterica) } \\
\text { Esterase, Est5 } \\
\text { (soil metagenome) } \\
\text { (N-terminus) } \\
\text { (Salmonella enterica) }\end{array}$ & $\begin{array}{l}\text { - Showed specific activity to their respective substrates but at varying efficiencies. } \\
\text { - Catalytic activity of immobilized } \beta \text {-gal catalyzing lactose conversion: } 62 \pm 7 \mu \mathrm{mol} / \mathrm{h} / \mathrm{mg} \text { of protein. } \\
\text { - Catalytic activity of soluble } \beta \text {-gal catalyzing lactose conversion: } 82 \pm 7 \mu \mathrm{mol} / \mathrm{h} / \mathrm{mg} \text { of protein. } \\
\text { - Catalytic activity of immobilized } \beta \text {-gal catalyzing o-nitrophenyl- } \beta \text {-galactoside (oNPG) conversion: } \\
4.2 \pm 0.17 \mu \mathrm{mol} / \mathrm{h} / \mathrm{mg} \text { of protein. } \\
\text { - Catalytic activity of soluble } \beta \text {-gal catalyzing oNPG conversion: } 3.9 \pm 0.11 \mu \mathrm{mol} / \mathrm{h} / \mathrm{mg} \text { of protein. } \\
\text { - Catalytic activity of immobilized } \beta \text {-gal catalyzing 4-methylumbelliferyl } \beta \text {-D-galactopyranoside (MUG) } \\
\text { conversion: } 3.2 \times 10^{6} \pm 1.8 \times 10^{5} \text { relative fluorescence unit (rfu)/min } / \mathrm{mg} \text { of protein. } \\
\text { - Catalytic activity of soluble } \beta \text {-gal catalyzing MUG conversion: } 5.0 \times 10^{6} \pm 1.7 \times 10^{4} \mathrm{rfu} / \mathrm{min} / \mathrm{mg} \text { of } \\
\text { protein. } \\
\text { - Catalytic activity of immobilized GldA catalyzing acetol conversion: } 1.1 \pm 0.2 \mu \mathrm{mol} / \mathrm{h} / \mathrm{mg} \text {. } \\
\text { - Catalytic activity of soluble GldA catalyzing acetol conversion: } 1.4 \pm 0.2 \mu \mathrm{mol} / \mathrm{h} / \mathrm{mg} \text {. } \\
\text { - Catalytic activity of immobilized GldA catalyzing methylglyoxal conversion: } 1.0 \pm 0.1 \mu \mathrm{mol} / \mathrm{h} / \mathrm{mg} \text {. } \\
\text { - Catalytic activity of soluble GldA catalyzing methylglyoxal conversion: } 2.1 \pm 0.4 \mu \mathrm{mol} / \mathrm{h} / \mathrm{mg} \text {. } \\
\text { - Catalytic activity of immobilized Est5 catalyzing 4-nitrophenyl butyrate }(\mathrm{pNP} \text {-butyrate) conversion: } \\
\text { - Catalytic activity of soluble Est5 catalyzing pNP-butyrate conversion: } 4.3 \pm 0.3 \mu \mathrm{mol} / \mathrm{h} / \mathrm{mg} \text {. }\end{array}$ & $\begin{array}{l}\text { - Enhanced pH stability } \\
\text { but not against thermal } \\
\text { stress. }\end{array}$ & $\begin{array}{l}\text { Jakobson et al. } \\
\text { 2016; Wagner } \\
\text { et al., 2017 }\end{array}$ \\
\hline $\begin{array}{l}\text { Salmonella enterica } \\
\text { Pdu bacterial } \\
\text { microcompartment } \\
\text { mutant SP subunit } \\
\text { O3-33 }\end{array}$ & $\begin{array}{l}\text { Alcohol dehydrogenase } \\
\text { D, AdhD } \\
\text { (Pyrococcus furiosus) } \\
\text { (N-terminus) } \\
\text { [E. coli BL21(DE3)] }\end{array}$ & $\begin{array}{l}\text { - Retained function but at decreased enzyme kinetic activity. } \\
\text { - } K_{m} \text { of immobilized AdhD for cofactor NAD+: } 140 \pm 20 \mu \mathrm{M} \text {. } \\
\text { - } K_{m} \text { of soluble AdhD for cofactor NAD }: 20 \pm 7 \mu \mathrm{M} \text {. } \\
\text { - } K_{m} \text { of immobilized AdhD for substrate 2,3-butanediol: } 140 \pm 10 \mathrm{mM} \text {. } \\
\text { - } K_{m} \text { of soluble AdhD for substrate 2,3-butanediol: } 38 \pm 8 \mathrm{mM} \text {. } \\
\text { - Turnover number }\left(k_{\text {cat }}\right) \text { of immobilized AdhD: } 0.046 \pm 0.002 \mathrm{~s}^{-1} \text {. } \\
\text { - } K_{c a t} \text { of soluble AdhD: } 0.088 \pm 0.009 \mathrm{~s}^{-1} \text {. } \\
\text { - Apparent } K_{m} \text { of immobilized AdhD for the elctrochemical activity: } 28 \pm 4 \mathrm{mM} \text {. } \\
\text { - Apparent } K_{m} \text { of soluble AdhD for the elctrochemical activity: } 27 \pm 3 \mathrm{mM} \text {. } \\
\text { - Apparent } k_{c a t} \text { of immobilized AdhD for the elctrochemical activity: } 0.0084 \pm 0.0001 \mathrm{~s}^{-1} \text {. } \\
\text { - Apparent } k_{c a t} \text { of soluble AdhD for the elctrochemical activity: } 0.0086 \pm 0.0002 \mathrm{~s}^{-1} \text {. }\end{array}$ & $\begin{array}{l}\text { - Doubled } \\
\text { electrochemical } \\
\text { operational stability. }\end{array}$ & $\begin{array}{l}\text { Bulutoglu et al., } \\
2019^{\wedge}\end{array}$ \\
\hline $\begin{array}{l}\text { Aquifex aeolicus } \\
\text { Lumazine synthase SP } \\
\text { subunit }\end{array}$ & $\begin{array}{l}\beta \text {-lactamase } \\
\text { (E. coli) } \\
\text { (C-terminus) } \\
{[\text { E. coli BL21(DE3)] }}\end{array}$ & • Enhanced catalytic activity catalyzing nitrocefin hydrolysis at specific configuration. & - N/A & $\begin{array}{l}\text { Choi et al., } \\
2018^{\wedge *}\end{array}$ \\
\hline $\begin{array}{l}\text { Thermotoga maritima } \\
\text { Ketohydroxyglutarate } \\
\text { aldolase SP subunit }\end{array}$ & $\begin{array}{l}(+)-\gamma \text {-Lactamase } \\
\text { (Microbacterium } \\
\text { hydrocarbonoxydans) } \\
\text { (N-terminus) } \\
{[\text { E. coli BL21(DE3)] }}\end{array}$ & $\begin{array}{l}\text { - } K_{m} \text { of immobilized }(+)-\gamma \text {-lactamase catalyzing Vince lactam hydrolysis: } 86 \pm 2.6 \mathrm{mM} \text {. } \\
-K_{m} \text { of soluble }(+)-\gamma \text {-lactamase catalyzing Vince lactam hydrolysis: } 120.4 \pm 7.2 \mathrm{mM} \text {. } \\
\text { - } \text { Kcat }_{\text {cat }} \text { of immobilized }(+)-\gamma \text {-lactamase catalyzing Vince lactam hydrolysis: } 12,830 \pm 164.5 \mathrm{~s}^{-1} \text {. } \\
\text { - } \text { Kcat }_{\text {cat }} \text { of soluble }(+)-\gamma \text {-lactamase catalyzing Vince lactam hydrolysis: } 20088 \pm 718 \mathrm{~s}^{-1} \text {. }\end{array}$ & $\begin{array}{l}\text { - Enhanced thermal } \\
\text { stability, higher } \\
\text { tolerance against } \\
\text { organic solvents, } \\
\text { proteolysis, and high } \\
\text { substrate } \\
\text { concentrations. }\end{array}$ & Li et al., 2018^ \\
\hline
\end{tabular}




\begin{tabular}{|c|c|c|c|c|}
\hline $\begin{array}{l}\text { Type of biological } \\
\text { scaffolds and their } \\
\text { anchoring motifs }\end{array}$ & $\begin{array}{l}\text { Target enzyme } \\
\text { (Origin) } \\
\text { (Gene fusion site) } \\
\text { (Production host) }\end{array}$ & Catalytic performance & Stability & References \\
\hline \multirow[t]{3}{*}{$\begin{array}{l}\text { Archaeoglobus fulgidus } \\
\text { Ferritin SP subunit }\end{array}$} & $\begin{array}{l}\text { Kemp eliminase } \\
\text { HG3.17 } \\
\text { (Thermoascus } \\
\text { aurantiacus) } \\
\text { (N-terminus) } \\
\text { [E. coli BL21-Gold } \\
\text { (DE3)] }\end{array}$ & 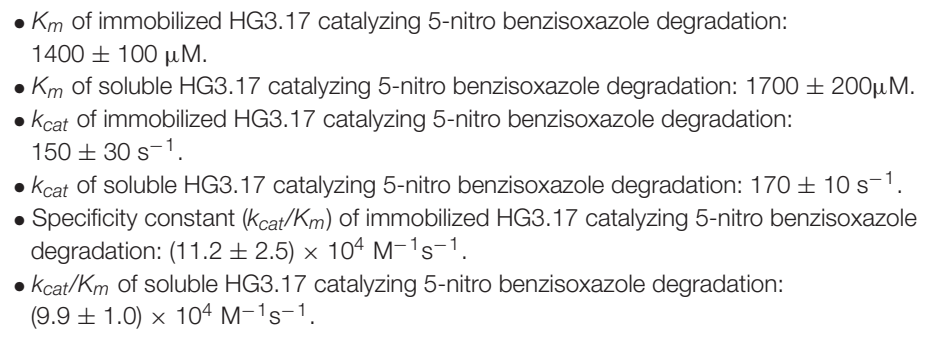 & $\begin{array}{l}\text { - Showed only partial } \\
\text { proteolytic protection } \\
\text { after incubation with } \\
\text { the blood plasma } \\
\text { protease factor Xa. } \\
\text { - Immobilized RA95.5-8F } \\
\text { showed enhanced } \\
\text { thermal stability. }\end{array}$ & $\begin{array}{l}\text { Tetter and } \\
\text { Hilvert, 2017^ }\end{array}$ \\
\hline & $\begin{array}{l}\text { Artificial retro-aldolase } \\
\text { RA95.5-8F } \\
\text { (Saccharolobus } \\
\text { solfataricus P2) } \\
\text { (C-terminus) } \\
\text { [E. coli BL21-Gold } \\
\text { (DE3)] }\end{array}$ & 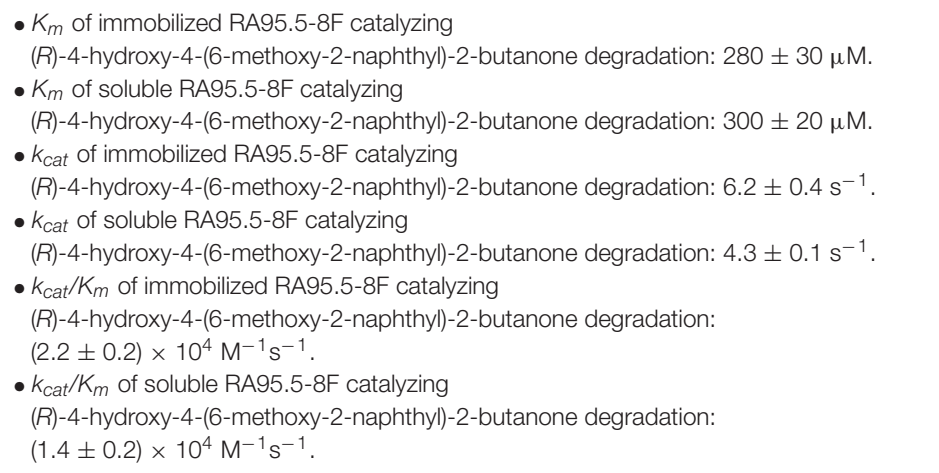 & & \\
\hline & $\begin{array}{l}\text { Carbonic anhydrase } 2 \\
\text { (Homo sapiens) } \\
\text { (N-terminus) } \\
\text { [E. coli BL21-Gold } \\
\text { (DE3)] }\end{array}$ & $\begin{array}{l}\text { - } k_{\text {cat }} / K_{m} \text { of immobilized carbonic anhydrase } 2 \text { catalyzing 4-nitrophenyl acetate } \\
\text { degradation: }(1.2 \pm 0.3) \times 10^{4} \mathrm{M}^{-1} \mathrm{~s}^{-1} \text {. } \\
\text { - } k_{\text {cat }} / K_{m} \text { of soluble carbonic anhydrase } 2 \text { catalyzing 4-nitrophenyl acetate degradation: } \\
(1.4 \pm 0.4) \times 10^{3} \mathrm{M}^{-1} \mathrm{~s}^{-1}\end{array}$ & & \\
\hline $\begin{array}{l}\text { Myxococcus xanthus } \\
\text { Encapsulin SP subunit }\end{array}$ & $\begin{array}{l}\text { Pyruvate } \\
\text { decarboxylase, Aro10p } \\
\text { (Saccharomyces } \\
\text { cerevisiae) } \\
\text { (C-terminus) } \\
\text { (Saccharomyces } \\
\text { cerevisiae PK2-1D) }\end{array}$ & $\begin{array}{l}\text { - Decarboxylation activity of immobilized Aro10p catalyzing 4-hydroxyphenylpyruvate } \\
\text { conversion to 4-hydroxyphenylacetaldehyde is consistent with its non-immobilized } \\
\text { counterpart. }\end{array}$ & $\begin{array}{l}\text { - Enhanced protection } \\
\text { against proteolytic } \\
\text { degradation. }\end{array}$ & $\begin{array}{l}\text { Lau et al., } \\
2018^{\wedge \star}\end{array}$ \\
\hline
\end{tabular}


TABLE 1 | Continued

\begin{tabular}{lll}
\hline $\begin{array}{l}\text { Type of biological } \\
\text { scaffolds and their }\end{array}$ & Target enzyme & Catalytic performance \\
anchoring motifs & (Origin) & \\
& (Gene fusion site) & \\
& (Production host)
\end{tabular}

\section{Extracellular membrane vesicles (EMVs)}

Outer membrane Endoglucanase CelA

vesicles (OMV) via

Pseudomonas syringae

INA5

Ice nucleation protein

InaV

Outer membrane

vesicles via E. coli outer

membrane porin

protein OmpA
(Clostridium

thermocellum)

(N-terminus)

(E. coli JC8031)

(Candida cellulolytica)

( $\mathrm{N}$-terminus)

(E. coli JC8031)

Endoglucanase CelG

(Candida cellulolytica)

(N-terminus)

(E. coli JC8031)

Organophosphorus

hydrolase, OpdA

(Flavobacterium sp

strain ATCC 27551)

(N-terminus)

(E. coli JC8031)

Phosphotriesterase

(Brevundimonas

diminuta)

(C-terminus)

[E. coli BL21(DE3)]
Exoglucanase CelE
- Artificial enzyme cascading system comprised of immobilized CelA, CelE, and CelG had

enhanced glucose production ( 23-fold higher) compared to its soluble counterpart.

- N/A

Park et al.

$2014^{\wedge *}$

- Enhanced paraoxon degradation rate with notable improvement in overall enzyme kinetics upon immobilization.

- $K_{m}$ of immobilized OpdA on OMV catalyzing paraoxon hydrolysis: $42.14 \pm 5.22 \mu \mathrm{M}$.

- $K_{m}$ of OpdA-OMV immobilized on microcrystalline cellulose catalyzing paraoxon hydrolysis:

$51.27 \pm 8.14 \mu \mathrm{M}$.

- $K_{m}$ of soluble OpdA catalyzing paraoxon hydrolysis: $47.95 \pm 9.36 \mu \mathrm{M}$.

- $k_{\text {cat }}$ of immobilized OpdA on OMV catalyzing paraoxon hydrolysis: $5716 \pm 379 \mathrm{~s}^{-1}$.

- $k_{\text {cat }}$ of OpdA-OMV immobilized on microcrystalline cellulose catalyzing paraoxon hydrolysis:

$5579 \pm 336 \mathrm{~s}^{-1}$

- $k_{\text {cat }}$ of soluble OpdA catalyzing paraoxon hydrolysis: $3513 \pm 216 \mathrm{~s}^{-1}$

- $k_{\text {cat }} / K_{m}$ of immobilized OpdA on OMV catalyzing paraoxon hydrolysis: $135.64 \pm 63.86$ $\mu \mathrm{M}^{-1} \mathrm{~s}^{-1}$

- $k_{c a t} / K_{m}$ of OpdA-OMV immobilized on microcrystalline cellulose catalyzing paraoxon hydrolysis

$108.82 \pm 18.48 \mu \mathrm{M}^{-1} \mathrm{~s}^{-1}$.

- $k_{\text {cat }} / K_{m}$ of soluble OpdA catalyzing paraoxon hydrolysis: $73.26 \pm 19.28 \mu \mathrm{M}^{-1} \mathrm{~s}^{-1}$

- Consistent with its soluble counterpart but showed enhanced activity in certain conditions.

- $K_{m}$ of immobilized phosphotriesterase catalyzing paraoxon hydrolysis: $47.3 \pm 3.1 \mu \mathrm{M}$.

- $K_{m}$ of soluble phosphotriesterase reported in the literature catalyzing paraoxon hydrolysis:

$90 \mu \mathrm{M}$.

- $k_{\text {cat }}$ of immobilized phosphotriesterase catalyzing paraoxon hydrolysis: $2088.7 \pm 47.8 \mathrm{~s}^{-1}$

- $k_{\text {cat }}$ of soluble phosphotriesterase reported in the literature catalyzing paraoxon hydrolysis:

$2400 s^{-1}$.

- $k_{c a t} / K_{m}$ of immobilized phosphotriesterase catalyzing paraoxon hydrolysis:

$(4.42 \pm 0.23) \times 10^{7} \mathrm{M}^{-1} \mathrm{~s}^{-1}$

- $\mathrm{K}_{\mathrm{cat}} / \mathrm{K}_{\mathrm{m}}$ of soluble phosphotriesterase reported in the literature catalyzing paraoxon hydrolysis: $2.7 \times 10^{7} \mathrm{M}^{-1} \mathrm{~s}^{-1}$
- Enhanced thermal and pH stability.

- Retained at least $83 \%$ of its initial activity after fifteen reaction cycles. Retained $\sim 20-30 \%$ of its initial activity after 40 days of storage.

- Less prone to enzyme inactivation by freezing, lyophilization.

- Challenging long-term storage and

environment conditions.
Su et al., 2017^

Alves et al.

2015, 2016,

2018^ 
TABLE 1 | Continued

\begin{tabular}{|c|c|c|c|c|}
\hline $\begin{array}{l}\text { Type of biological } \\
\text { scaffolds and their } \\
\text { anchoring motifs }\end{array}$ & $\begin{array}{l}\text { Target enzyme } \\
\text { (Origin) } \\
\text { (Gene fusion site) } \\
\text { (Production host) }\end{array}$ & Catalytic performance & Stability & References \\
\hline \multicolumn{5}{|l|}{ Magnetosomes } \\
\hline \multirow[t]{2}{*}{$\begin{array}{l}\text { Magnetosome } \\
\text { membrane protein } \\
\text { MamC }\end{array}$} & $\begin{array}{l}\text { Organophosphohydrolase, } \\
\text { OpdA } \\
\text { (Flavobacterium sp. } \\
\text { ATCC 27551) } \\
\text { (Magnetospirillum } \\
\text { magneticum AMB-1) }\end{array}$ & $\begin{array}{l}-K_{m} \text { of immobilized OpdA catalyzing ethyl-paraoxon hydrolysis: } 58 \pm 2.5 \mu \mathrm{M} \text {. } \\
-K_{m} \text { of soluble OpdA catalyzing ethyl-paraoxon hydrolysis: } 43 \pm 1.8 \mu \mathrm{M} \text {. } \\
\text { - } K_{\text {cat }} \text { of immobilized OpdA catalyzing ethyl-paraoxon hydrolysis: } 151 \pm 6 \mathrm{~s}^{-1} \text {. } \\
\text { - } \text { Kcat }_{\text {cat }} \text { of soluble OpdA catalyzing ethyl-paraoxon hydrolysis: } 314 \pm 13 \mathrm{~s}^{-1} \text {. }\end{array}$ & $\begin{array}{l}\text { - Stable over six reaction } \\
\text { cycles. }\end{array}$ & Ginet et al., $2011^{\wedge}$ \\
\hline & $\begin{array}{l}\beta \text {-glucuronidase } \\
\text { (E. coli) } \\
\text { (C-terminus) } \\
\text { (Magnetospirillum } \\
\text { gryphiswaldense) }\end{array}$ & $\begin{array}{l}\text { - Km of immobilized } \beta \text {-glucuronidase catalyzing } p \text {-nitrophenyl- } \beta \text {-D-glucuronide } \\
\text { hydrolysis: } 0.17 \times 10^{-3}-0.18 \times 10^{-3} \mathrm{M} \text {. } \\
\text { - } K_{m} \text { of soluble } \beta \text {-glucuronidase catalyzing } p \text {-nitrophenyl- } \beta \text {-D-glucuronide hydrolysis: } \\
0.28 \times 10^{-3} \mathrm{M} \text {. } \\
\text { - Specific activity of immobilized } \beta \text {-glucuronidase catalyzing } \\
p \text {-nitrophenyl- } \beta \text {-D-glucuronide hydrolysis: } 15.1-16.3 \mathrm{U} / \mathrm{mg} \text { of enzyme. } \\
\text { - Specific activity of soluble } \beta \text {-glucuronidase catalyzing } p \text {-nitrophenyl- } \beta \text {-D-glucuronide } \\
\text { hydrolysis: } 12.7 \mathrm{U} / \mathrm{mg} \text { of enzyme. }\end{array}$ & $\begin{array}{l}\text { - Retained at least } \sim 75 \% \\
\text { of its initial activity after } \\
10 \text { reaction cycles. }\end{array}$ & $\begin{array}{l}\text { Mickoleit and Schüler, } \\
2018\end{array}$ \\
\hline $\begin{array}{l}\text { Magnetosome } \\
\text { membrane protein } \\
\text { Mms13 }\end{array}$ & $\begin{array}{l}\text { Endoglucanase A } \\
\text { (Clostridium } \\
\text { thermocellum) } \\
\text { (C-terminus) } \\
\text { (Magnetospirillum } \\
\text { magneticum AMB-1) } \\
\beta \text {-Glucosidase } \\
\text { (Clostridium } \\
\text { thermocellum) } \\
\text { (C-terminus) } \\
\text { (Magnetospirillum } \\
\text { magneticum AMB-1) }\end{array}$ & $\begin{array}{l}\text { - Artificial enzyme cascading system comprised of these two enzymes showed } \\
\text { catalytic activity catalyzing the hydrolysis of carboxymethyl cellulose and Avicel. } \\
\text { - Co-immobilization of endoglucanase A and } \beta \text { - glucosidase on magnetosomes } \\
\text { showed enhanced catalytic activity catalyzing the hydrolysis of carboxymethyl } \\
\text { cellulose when compared to the suspension mixture of endoglucanase A } \\
\text { immobilized magnetosomes and } \beta \text {-glucosidase immobilized magnetosomes. }\end{array}$ & $\begin{array}{l}\text { - Retained at least } \sim 70 \% \\
\text { of its initial activity after } \\
\text { five reaction cycles. }\end{array}$ & Honda et al., $2015 a^{\wedge \star}$ \\
\hline
\end{tabular}

$\wedge^{\wedge}$ Specific and/or catalytic activities are not mentioned in the reference. *Kinetic parameters are not mentioned in the reference. 
Hay et al., 2015; Parlane et al., 2016a). Assembly of immobilized multiprotein complexes enables multi-enzymatic cascade systems with superior catalytic performance as recently reviewed (Hwang and Lee, 2019). Flexible, rigid, and cleavable peptide linkers, such as intein peptide pairs (Du and Rehm, 2017) and LPXTG cleavage sites (sortase A-mediated hydrolysis/ligation) (Du and Rehm, 2018), can be incorporated between the protein functions and PAPs to mediate release of pure target protein (Hay et al., 2014; Rehm et al., 2018). However, underlying molecular mechanisms of PHA particle formation still remain unknown, which intrinsically limits control of their physicochemical properties. For example, a few studies reported that fusing different proteins to $\mathrm{PhaC}$ influences the PHA production yield over biomass, particle size distribution, surface charges, and purity of the target protein (Rubio-Reyes et al., 2016; GonzalezMiro et al., 2018a,b; Wong and Rehm, 2018). Decorating PHA particles with proteins using PhaC synthase as an anchoring domain can also cause varying distribution and density of respective proteins on the PHA particles (Hay et al., 2015; Wong and Rehm, 2018). Hooks et al. (2013) also pointed out that displaying $\mathrm{N}$-acetylneuraminic acid aldolase (NanA) from E. coli on PHA particles through $\mathrm{N}$ - and C-terminal fusion of PhaC resulted in varying catalytic performance (Hooks et al., 2013). Moreover, similar findings were reported for phasins where fusion of different foreign polypeptides to the BioF tag
(PHA-binding domain of PhaF) might have contributed to inconsistency of the physical adsorption function of the BioFtagged enzyme to the PHA particle surface (Bello-Gil et al., 2018a). A brief comparison of the PHA particle technology with other biological assemblies, detailing their advantages and limitations, is provided in Table 2 .

To circumvent these drawbacks regarding the utilization of the PHA particle technology, our group merged the PhaC fusion technology with the SpyTag/SpyCatcher chemistry (Zakeri et al., 2012), which enable better control over production yields and physicochemical properties (Figure 3A) (Wong and Rehm, 2018). We successfully showed that the SpyTagged proteins could ligate to the SpyCatcher-PhaC coated PHA particles in vitro, and controlled multifunctionality of PHA particles could be achieved using a sequential immobilization strategy. This approach requires separate and more laborious production of enzymes and scaffold but offers more control over surface coverage and orientation/ratios of the attached proteins. Consistency of the particle size and surface charge of functionalized SpyCatcher-coated PHA particles were observed. Function and conformational stability of the ligated proteins were retained or enhanced (Wong and Rehm, 2018). Recently this approach was expanded by developing streamlined processes exploiting the specificity of the SpyTag/SpyCatcher mediated ligation for efficient and cost-effective modular functionalization

TABLE 2 | Comparison of PHA particle technology with other biological supramolecular assemblies.

\begin{tabular}{|c|c|c|}
\hline & Advantages & Limitations \\
\hline Polyhydroxyalkanoates (PHAs) & $\begin{array}{l}\text { - Scalable particle production and able to offer better } \\
\text { production yields over biomass } \\
\text { - Facile particle functionalization and isolation steps } \\
\text { - Structurally very stable } \\
\text { - Can be manufactured in a range of recombinant } \\
\text { expression systems } \\
\text { - Biodegradable } \\
\text { - Enhanced shelf-life }\end{array}$ & $\begin{array}{l}\text { - Poor controllability on the physicochemical } \\
\text { properties of the particles (e.g., particle size, size } \\
\text { distribution, surface charge) - polydisperse and } \\
\text { tend to aggregate } \\
\text { - Concentration and the function of enzymes } \\
\text { localized on the particles, and particle production } \\
\text { yield dependent on the folding status of the } \\
\text { recombinant fusion proteins }\end{array}$ \\
\hline $\begin{array}{l}\text { Protein-based particles } \\
\text { - Virus-like particles (VLPs) } \\
\text { - Enzyme-derived nanoparticles (EZPs) }\end{array}$ & $\begin{array}{l}\text { - Highly programmable physicochemical properties of } \\
\text { particles (e.g., particle size, size distribution, surface } \\
\text { charge) } \\
\text { - Multiple modes of immobilization - tethered within } \\
\text { and/or on the surface of particles, and between the } \\
\text { CP/SP subunits } \\
\text { - Can be manufactured in a range of recombinant } \\
\text { expression systems } \\
\text { - Biodegradable }\end{array}$ & $\begin{array}{l}\text { - Genetic alteration of CP/SP subunits could trigger } \\
\text { structural instability of these scaffolding platforms } \\
\text { - Could lead to misfolding of the genetically fused } \\
\text { enzymes, especially large domains due to steric } \\
\text { hindrance } \\
\text { - Labor intensive fabrication processes } \\
\text { - Scalability issues } \\
\text { - Space available to immobilize functional moieties is } \\
\text { limited by the size of the scaffold itself }\end{array}$ \\
\hline Extracellular membrane vesicles (EMVs) & $\begin{array}{l}\text { - Easy decoration of vesicles } \\
\text { - Enzymes of interest can be appended on the surface } \\
\text { or within the vesicles } \\
\text { - Can be manufactured in a range of recombinant } \\
\text { expression systems } \\
\text { - Biodegradable }\end{array}$ & $\begin{array}{l}\text { - Poor particle programmability due to the lack of } \\
\text { knowledge on the exact assembly mechanism of } \\
\text { membrane vesicles } \\
\text { - Large-scale consistent production could be difficult } \\
\text { - Laborious and expensive isolation procedures }\end{array}$ \\
\hline Magnetosomes & $\begin{array}{l}\text { - Unique magnetic properties of magnetosomes could } \\
\text { be advantageous in some applications (e.g., } \\
\text { magnetically driven solid-liquid separation for re-use) } \\
\text { - Consistent particle size, particle distribution, and } \\
\text { architecture }\end{array}$ & $\begin{array}{l}\text { - Tedious cloning steps and limited design space } \\
\text { available for extensive alterations in vivo due to } \\
\text { potential cell toxicity } \\
\text { - Poor controllability in altering the magnetic } \\
\text { properties - influenced by the specificity of } \\
\text { magnetotatic bacteria } \\
\text { - Magnetotatic bacteria are difficult to } \\
\text { grow-prolonged production time and low } \\
\text { production yields }\end{array}$ \\
\hline
\end{tabular}


A

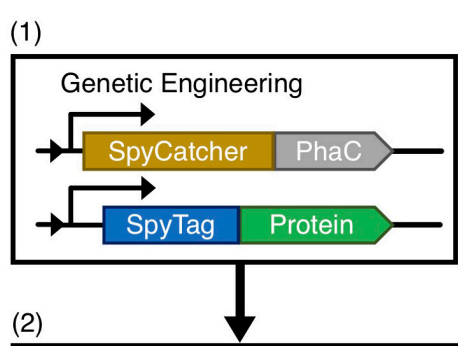

SpyCatcher-coated PHA particles only

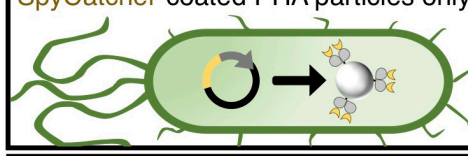

SpyTagged proteins only

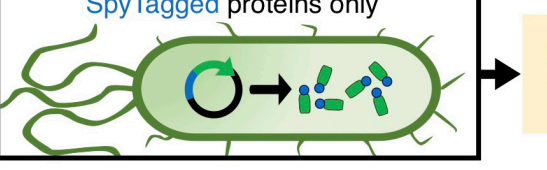

SpyCatcher SpyTag

(Lys) (Asp)

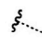<smiles>CCCCCCC</smiles><smiles>CCC(=O)CC</smiles>

$\downarrow$ Amidation

(3)<smiles>CCCCCCNC(=O)CCI</smiles>

Isolated SpyCatchercoated PHA particles

Purified SpyTagged proteins

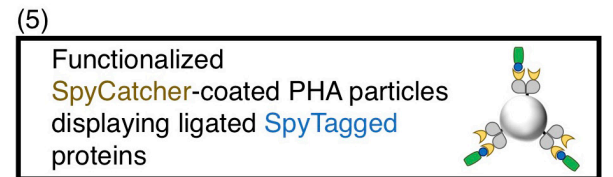

(4)

Mixture of purified components in vitro
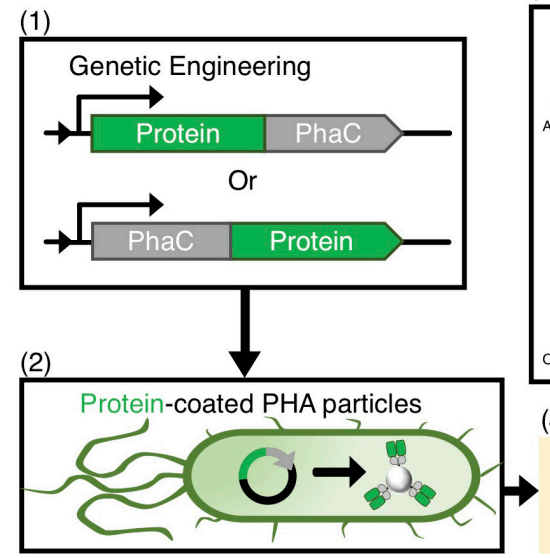

(4)

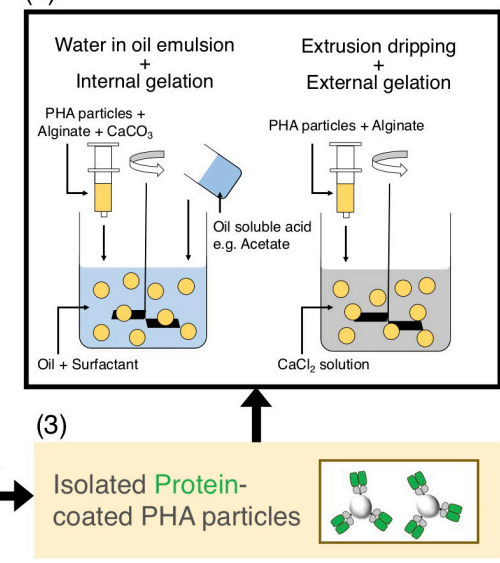

(5)

Functionalized Protein-coated PHA particles + alginate composite material

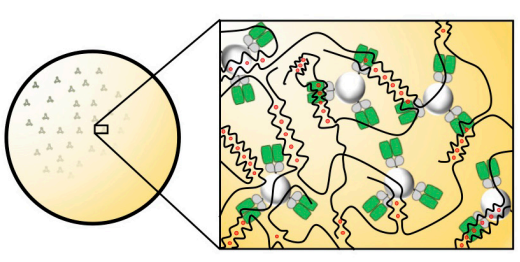

Entrapment of PHA particles within alginate crosslinked polymer networks

$\sqrt{ }$ Alginate

- Crosslinker e.g., $\mathrm{Ca}^{2+}$

$\hat{\imath}$ Crosslinked structure "Egg-Box"

FIGURE 3 | (A) Schematic overview of biosynthesis and modular functionalization of SpyCatcher-coated PHA particles. (B) Schematic illustration of manufacturing of functionalized PHA particles and subsequent fabrication of alginate-PHA composite materials.

(Wong et al., 2020). Overall, PHA particles seem to provide a versatile platform for in vivo enzyme immobilization, providing competitive advantages over other biological scaffolds (Table 2). The recent crystal structures, 3D-reconstructed, and homology models of several key PAPs, including Cupriavidus necator $\mathrm{PhaC}$ and some $\mathrm{PhaPs}$ will further inform protein engineering for efficient immobilization of enzymes (Wittenborn et al., 2016; Zhao et al., 2016, 2017; Kim J. et al., 2017; Kim Y. J. et al., 2017).

\section{Use of Enzyme-Coated PHA Particles in Continuous-Flow Bioprocessing}

Immobilized enzymes are widely considered for continuous flow processing toward the synthesis of high-value chemicals (Lee et al., 2015; Contente et al., 2017; Peschke et al., 2017; Döbber et al., 2018; van den Biggelaar et al., 2019).
Continuous production of fine chemicals has the potential to accelerate biocatalytic transformations due to enhanced heat and mass transfer between immobilized enzymes and their substrates under flow conditions. The improvement in mass transfer allows the cost-effective miniaturized design of process equipment that ultimately could lead to precise process control and better production yield. Continuous bioprocessing could simplify downstream processing and permit the constant removal of products, such as processes limited by a thermodynamic equilibrium (Tamborini et al., 2018; Thompson et al., 2018). The physical format of the immobilized enzymes needs to be compatible with the continuous-flow process, such as tangential-flow filtration and packed bed/fluidized bed systems (Truppo, 2017).

The lack of uniformity and/or the non-porous properties may restrict the utility of enzyme-coated PHA particles for industrial continuous bioprocesses. Apart from the inherent 
inconsistency of the PHA particles as outlined above, particulate carriers $(<1 \mu \mathrm{m})$ are often prone to aggregation under various environmental conditions (e.g., $\mathrm{pH}$, temperature, and ionic strength), which impairs substrate access to the enzymes (Zhang, 2014; Thoniyot et al., 2015) and which could adversely affect their performance in continuous flow processes. The non-porous nature of PHA particles (Hooks et al., 2014) and their tendency to aggregate will cause extensive back-pressure in flow-through applications (Ogura and Rehm, 2019). One innovative solution to overcome these issues is to encapsulate the functionalized PHA particles into a porous hydrogel matrix for efficient integration of enzyme-coated PHA particles into continuousflow bioprocesses. We recently described an innovative approach that encapsulates functionalized PHA particles within a highly amenable anionic polysaccharide, alginate. The particle-hydrogel composite material was fabricated using the ionotropic gelation method with calcium ion as the crosslinker (Figure 3B) (Ogura and Rehm, 2019). Interestingly, the porosity of the alginate microsphere encapsulating functional protein-coated PHA particles could be controlled by $\mathrm{pH}$ during the fabrication process, showing the flexibility of this approach. The various functional protein-coated PHA particles encapsulated within alginate microspheres showed either retained (e.g., organophosphorus hydrolase) or enhanced (e.g., immunoglobulin G-binding ZZ domain) activities in both batch and flow-through mode suggesting suitability for industrial applications (Ogura and Rehm, 2019).

\section{Potential Industrial Applications of the PHA Particle Technology}

There is a widespread agreement that enzyme mediated bioprocesses are environmentally benign as, for example, they reduce consumption of raw materials and energy, while generally able to maintain low levels of waste generation than the traditional non-enzymatic processes (DiCosimo et al., 2013). Implementation of enzymes in large-scale manufacturing could reduce the greenhouse gas emissions when compared to the traditional non-enzymatic processes (Jegannathan and Nielsen, 2013). Therefore, due to the disadvantages in using industrially relevant enzymes in soluble form as mentioned, direct attachment of these enzymes to solid scaffolds, including PHAs, emerged as one of the commercially viable solutions. The advent of PHA particle technology as a generic scaffolding platform for immobilization of enzymes has opened up new routes in developing next-generation catalytic materials for sustainable bioprocessing. We have summarized the recent proof-of-concept demonstrations of the PHA particle technology for industrial applications reported by our group and others (Table 1). Task-specific designer PHA particles can be biosynthesized to serve different industrial applications including the manufacture of commodity chemicals, food products, active pharmaceutical ingredients, and cosmetic chemicals (Abdelraheem et al., 2019). Furthermore, the PHA particle technology can be implemented as a bioremediation tool for the treatment of industrial waste effluents and agricultural pollutants (Sharma et al., 2018).
Since bulk chemicals, such as, e.g., commodity chemicals and food products, are produced at ton scale, high catalytic turnover and ease of reusability of biocatalysts are required for economic feasibility (DiCosimo et al., 2013; Basso and Serban, 2019). Biocatalysts also need to be accessible at low cost and need to be highly stable (Basso and Serban, 2019). On the contrary, different factors need to be considered for production of fine chemicals, such as, e.g., active pharmaceutical ingredients, and cosmetic chemicals, as it is often associated with lower production volumes (e.g., hundreds of kilograms) but higher production yields (Basso and Serban, 2019). More expensive biocatalysts could be considered while the synthesis of these high-value products requires a certain degree of regioselectivity, enantioselectivity, and chemoselectivity (Goldsmith and Tawfik, 2012; Palomo and Guisan, 2012). In addition, the successful implementation of immobilized biocatalysts such as enzyme-coated PHA particles in continuous bioprocessing will be advantageous for production of fine chemicals due to the need for precise process control to achieve the required product quality (Thompson et al., 2018). For bioremediation application, the PHA particle technology offers advantages such as biodegradability of the non-toxic natural PHA scaffold (Ong et al., 2017; Koller, 2018). In recent years, the release of nanoparticles to the environment has sparked some concerns by the research community (Nguyen et al., 2011; Bundschuh et al., 2018).

Given the encouraging proof-of-concept results adapting the PHA particle technology for development of immobilized enzymes for uses in the food industry (Moldes et al., 2004; Rasiah and Rehm, 2009; Ran et al., 2017, 2019), production of commodity chemicals (Jahns and Rehm, 2015; Yang et al., 2015; Seo et al., 2016), production of fine chemicals (Hooks et al., 2013; Chen S. Y. et al., 2014; Tan et al., 2019), and bioremediation (Robins et al., 2013; Hooks and Rehm, 2015; Li et al., 2019), it is anticipated that research prototypes will be developed into industrial products.

\section{CONCLUSION AND FUTURE PERSPECTIVES}

Here, we reviewed the advances in the development of several promising biological supramolecular assemblies suitable for in vivo enzyme immobilization. We then compared the PHA particle technology with the other scaffolding platforms and discussed innovative strategies to address the challenges associated with developing enzyme-coated PHA particles for industrial applications. Immobilized enzymes exhibit distinct advantages over soluble enzymes, including enhanced stability, improved catalytic performance, reusability, and facilitated product purification. The emergence of biologically inspired particulate carriers has offered promising scaffolding platforms for one-pot in vivo enzyme immobilization. Though significant progress has been made to date, numerous challenges, such as high production costs and lack of control over a range of physicochemical properties, need to be tackled to advance these immobilization technologies beyond the proof-of-concept. 
As the field of synthetic biology continues to expand rapidly, a more profound understanding of the underlying molecular mechanisms of particle assembly in vivo will further inform the rational design of assembled enzyme-carrier systems. The elucidation of these biological processes in vivo informs strategies to control several aspects as, for instance, simultaneous PHA particle production and functionalization rational molecular engineering approaches. Such customizable features would allow the creation of, for example, application-specific designer PHA particles for a variety of operating environments. We expect that these remarkable advances can also lay the foundation for the development of monodisperse PHA particles of controllable and reproducible structure and size with programmable surface properties, such as enzyme density/exposure and surface charge. Furthermore, implementing innovative strategies, such as the concept of modularity, fabrication of particle-hydrogel composite materials, and integrated multifunctionality, should increasingly enable implementation in industrial flow-through processes. The development of robust enzyme-carrier systems with porous structures will be critical to ensure implementation for cost-effective continuous biocatalytic conversion and synthesis reactions.

The versatile PHA particle technology offers avenues to immobilize a range of industrially relevant enzymes for development of the next-generation biocatalytic processes. However, the successful "bench-to-factory" translation still requires rigorous optimization and validation to meet industry standards. Additionally, perception barriers as, for instance, the traditional way of thinking and the limited knowledge on sustainable bioprocessing, especially among the manufacturers and regulatory authorities, could hinder the

\section{REFERENCES}

Abdelraheem, E. M. M., Busch, H., Hanefeld, U., and Tonin, F. (2019). Biocatalysis explained: from pharmaceutical to bulk chemical production. React. Chem. Eng. 4, 1878-1894. doi: 10.1039/C9RE00301K

Alves, N. J., Moore, M., Johnson, B. J., Dean, S. N., Turner, K. B., Medintz, I. L., et al. (2018). Environmental decontamination of a chemical warfare simulant utilizing a membrane vesicle-encapsulated phosphotriesterase. ACS Appl. Mater. Interfaces 10, 15712-15719. doi: 10.1021/acsami.8b02717

Alves, N. J., Turner, K. B., Daniele, M. A., Oh, E., Medintz, I. L., and Walper, S. A. (2015). Bacterial nanobioreactors-directing enzyme packaging into bacterial outer membrane vesicles. ACS Appl. Mater. Interfaces 7, 24963-24972. doi: 10.1021/acsami.5b08811

Alves, N. J., Turner, K. B., Medintz, I. L., and Walper, S. A. (2016). Protecting enzymatic function through directed packaging into bacterial outer membrane vesicles. Sci. Rep. 6:24866. doi: 10.1038/srep24866

Arakaki, A., Nakazawa, H., Nemoto, M., Mori, T., and Matsunaga, T. (2008). Formation of magnetite by bacteria and its application. J. R. Soc. Interface 5, 977-999. doi: 10.1098/rsif.2008.0170

Arosio, P., Ingrassia, R., and Cavadini, P. (2009). Ferritins: a family of molecules for iron storage, antioxidation and more. Biochim. Biophys. Acta 1790, 589-599. doi: 10.1016/j.bbagen.2008.09.004

Arrighetti, N., Corbo, C., Evangelopoulos, M., Pastò, A., Zuco, V., and Tasciotti, E. (2019). Exosome-like nanovectors for drug delivery in cancer. Curr. Med. Chem. 26, 6132-6148. doi: 10.2174/0929867325666180831150259

Azuma, Y., Edwardson, T. G., and Hilvert, D. (2018). Tailoring lumazine synthase assemblies for bionanotechnology. Chem. Soc. Rev. 47, 3543-3557. doi: 10.1039/ C8CS00154E application of these new catalytic materials. Therefore, bridging interdisciplinary boundaries between researchers from the field of molecular biology, chemical engineering, chemistry, and material science should be encouraged. It is critical to integrate diverse methodologies and strategies to further advance in vivo enzyme immobilization technologies such as the PHA particle technology.

\section{AUTHOR CONTRIBUTIONS}

KO wrote section "Enzyme Immobilization for Industrial Applications" of the manuscript. SC wrote section "Utilization of Various Supramolecular Assemblies as Enzyme Immobilization Supports" of the manuscript. JW wrote sections "Biological Supramolecular Assemblies as Biocatalyst Supports" and "Comparative Analysis of in vivo Immobilization Strategies" of the manuscript. BR provided critical input in regard to structure, content, and language of the manuscript. All authors provided critical feedback and approved the final version of the manuscript.

\section{FUNDING}

This work was supported by the MacDiarmid Institute of Advanced Materials and Nanotechnology (New Zealand), the School of Fundamental Sciences, Massey University (New Zealand), the School of Environment and Science, Griffith University (Australia), and the Centre for Cell Factories and Biopolymers, Griffith University (Australia).

Bakhshi, P. K., Bain, J., Gul, M. O., Stride, E., Edirisinghe, M., and Staniland, S. S. (2016). Manufacturing man-Made magnetosomes: high-throughput in situ synthesis of biomimetic magnetite loaded nanovesicles. Macromol. Biosci. 16, 1555-1561. doi: 10.1002/mabi.201600181

Bale, J. B., Gonen, S., Liu, Y., Sheffler, W., Ellis, D., Thomas, C., et al. (2016). Accurate design of megadalton-scale two-component icosahedral protein complexes. Science 353, 389-394. doi: 10.1126/science.aaf8818

Basso, A., and Serban, S. (2019). Industrial applications of immobilized enzymesA review. Mol. Catal. 479:110607. doi: 10.1016/j.mcat.2019.110607

Bello-Gil, D., Maestro, B., Fonseca, J., Dinjaski, N., Prieto, M. A., and Sanz, J. M. (2018a). Poly-3-hydroxybutyrate functionalization with BioF-tagged recombinant proteins. Appl. Environ. Microbiol. 84:e02595-17. doi: 10.1128/ AEM.02595-17

Bello-Gil, D., Roig-Molina, E., Fonseca, J., Sarmiento-Ferrández, M. D., Ferrándiz, M., Franco, E., et al. (2018b). An enzymatic system for decolorization of wastewater dyes using immobilized CueO laccase-like multicopper oxidase on poly-3-hydroxybutyrate. Microb. Biotechnol. 11, 881-892. doi: 10.1111/17517915.13287

Bernal, C., Rodríguez, K., and Martínez, R. (2018). Integrating enzyme immobilization and protein engineering: an alternative path for the development of novel and improved industrial biocatalysts. Biotechnol. Adv. 36, 1470-1480. doi: 10.1016/j.biotechadv.2018.06.002

Brasch, M., Putri, R. M., De Ruiter, M. V., Luque, D., Koay, M. S., Castoìn, J. R., et al. (2017). Assembling enzymatic cascade pathways inside virus-based nanocages using dual-tasking nucleic acid tags. J. Am. Chem. Soc. 139, 1512-1519. doi: 10.1021/jacs.6b10948

Bruce, T. F., Slonecki, T. J., Wang, L., Huang, S., Powell, R. R., and Marcus, R. K. (2019). Exosome isolation and purification via hydrophobic interaction 
chromatography using a polyester, capillary-channeled polymer fiber phase. Electrophoresis 40, 571-581. doi: 10.1002/elps.201800417

Brune, K. D., Leneghan, D. B., Brian, I. J., Ishizuka, A. S., Bachmann, M. F., Draper, S. J., et al. (2016). Plug-and-display: decoration of virus-like particles via isopeptide bonds for modular immunization. Sci. Rep. 6:19234. doi: 10.1038/ srep19234

Bulutoglu, B., Macazo, F. C., Bale, J., King, N., Baker, D., Minteer, S. D., et al. (2019). Multimerization of an alcohol dehydrogenase by fusion to a designed selfassembling protein results in enhanced bioelectrocatalytic operational stability. ACS Appl. Mater. Interfaces 11, 20022-20028. doi: 10.1021/acsami.9b04256

Bundschuh, M., Filser, J., Lüderwald, S., Mckee, M. S., Metreveli, G., Schaumann, G. E., et al. (2018). Nanoparticles in the environment: where do we come from, where do we go to? Environ. Sci. Eur. 30:6. doi: 10.1186/s12302-018-0132-6

Burkova, E. E., Dmitrenok, P. S., Bulgakov, D. V., Vlassov, V. V., Ryabchikova, E. I., and Nevinsky, G. A. (2018). Exosomes from human placenta purified by affinity chromatography on sepharose bearing immobilized antibodies against CD81 tetraspanin contain many peptides and small proteins. IUBMB Life 70, 1144-1155. doi: 10.1002/iub.1928

Bustos-Jaimes, I., Soto-Román, R. A., Gutiérrez-Landa, I. A., Valadez-García, J., and Segovia-Trinidad, C. L. (2017). Construction of protein-functionalized virus-like particles of parvovirus B19. J. Biotechnol. 263, 55-63. doi: 10.1016/ j.jbiotec.2017.09.014

Campisano, A., Overhage, J., and Rehm, B. H. (2008). The polyhydroxyalkanoate biosynthesis genes are differentially regulated in planktonic-and biofilm-grown Pseudomonas aeruginosa. J. Biotechnol. 133, 442-452. doi: 10.1016/j.jbiotec. 2007.11.007

Cao, X., Li, Y., Zhang, Z., Yu, J., Qian, J., and Liu, S. (2012). Catalytic activity and stability of glucose oxidase/horseradish peroxidase co-confined in macroporous silica foam. Analyst 137, 5785-5791. doi: 10.1039/c2an36237f

Cayetano-Cruz, M., Coffeen, C. F., Valadez-García, J., Montiel, C., and BustosJaimes, I. (2018). Decoration of virus-like particles with an enzymatic activity of biomedical interest. Virus Res. 255, 1-9. doi: 10.1016/j.virusres.2018.06.014

Chapman, J., Ismail, A., and Dinu, C. (2018). Industrial applications of enzymes: recent advances, techniques, and outlooks. Catalysts 8:238. doi: 10.3390/ catal 8060238

Charlton Hume, H. K., Vidigal, J., Carrondo, M. J., Middelberg, A. P., Roldão, A., and Lua, L. H. (2019). Synthetic biology for bioengineering virus-like particle vaccines. Biotechnol. Bioeng. 116, 919-935. doi: 10.1002/bit.26890

Chen, Q., Sun, Q., Molino, N. M., Wang, S.-W., Boder, E. T., and Chen, W. (2015). Sortase A-mediated multi-functionalization of protein nanoparticles. Chem. Commun. 51, 12107-12110. doi: 10.1039/c5cc03769g

Chen, S., Parlane, N. A., Lee, J., Wedlock, D. N., Buddle, B. M., and Rehm, B. H. (2014). New skin test for detection of bovine tuberculosis on the basis of antigen-displaying polyester inclusions produced by recombinant Escherichia coli. Appl. Environ. Microbiol. 80, 2526-2535. doi: 10.1128/AEM.04168-13

Chen, S. Y., Chien, Y.-W., and Chao, Y.-P. (2014). In vivo immobilization of D-hydantoinase in Escherichia coli. J. Biosci. Bioeng. 118, 78-81. doi: 10.1016/ j.jbiosc.2013.12.020

Choi, H., Choi, B., Kim, G. J., Kim, H. U., Kim, H., Jung, H. S., et al. (2018). Fabrication of nanoreaction clusters with dual-functionalized protein cage nanobuilding blocks. Small 14:1801488. doi: 10.1002/smll.201801488

Cipolatti, E. P., Manoel, E. A., Fernandez-Lafuente, R., and Freire, D. M. G. (2017). Support engineering: relation between development of new supports for immobilization of lipases and their applications. Biotechnol. Res. Innov. 1, 26-34. doi: 10.1016/j.biori.2017.01.004

Cocucci, E., and Meldolesi, J. (2015). Ectosomes and exosomes: shedding the confusion between extracellular vesicles. Trends Cell Biol. 25, 364-372. doi: $10.1016 /$ j.tcb.2015.01.004

Collins, B. S. (2011). Gram-negative outer membrane vesicles in vaccine development. Discov. Med. 12, 7-15.

Contente, M. L., Dall'oglio, F., Tamborini, L., Molinari, F., and Paradisi, F. (2017). Highly efficient oxidation of amines to aldehydes with flow-based biocatalysis. Chem CatChem 9, 3843-3848. doi: 10.1002/cctc.201701147

Diaz, D., Care, A., and Sunna, A. (2018). Bioengineering strategies for proteinbased nanoparticles. Genes 9:370. doi: 10.3390/genes9070370

DiCosimo, R., Mcauliffe, J., Poulose, A. J., and Bohlmann, G. (2013). Industrial use of immobilized enzymes. Chem. Soc. Rev. 42, 6437-6474. doi: 10.1039/ c3cs35506c
Dieudonné, A., Pignol, D., and Prévéral, S. (2019). Magnetosomes: biogenic iron nanoparticles produced by environmental bacteria. Appl. Microbiol. Biotechnol. 103, 3637-3649. doi: 10.1007/s00253-019-09728-9

Döbber, J., Gerlach, T., Offermann, H., Rother, D., and Pohl, M. (2018). Closing the gap for efficient immobilization of biocatalysts in continuous processes: HaloTag ${ }^{\mathrm{TM}}$ fusion enzymes for a continuous enzymatic cascade towards a vicinal chiral diol. Green Chem. 20, 544-552. doi: 10.1039/c7gc03225k

$\mathrm{Du}$, J., and Rehm, B. H. (2017). Purification of target proteins from intracellular inclusions mediated by intein cleavable polyhydroxyalkanoate synthase fusions. Microb. Cell Fact. 16:184. doi: 10.1186/s12934-017-0799-1

Du, J., and Rehm, B. H. (2018). Purification of therapeutic proteins mediated by in vivo polyester immobilized sortase. Biotechnol. Lett. 40, 369-373. doi: 10.1007/s10529-017-2473-4

Faccio, G. (2018). From protein features to sensing surfaces. Sensors 18:E1204. doi: $10.3390 /$ s18041204

Fernandez-Lopez, L., Pedrero, S. G., Lopez-Carrobles, N., Gorines, B. C., VirgenOrtíz, J. J., and Fernandez-Lafuente, R. (2017). Effect of protein load on stability of immobilized enzymes. Enzyme Microb. Technol. 98, 18-25. doi: 10.1016/j. enzmictec.2016.12.002

Fu, J., Liu, M., Liu, Y., Woodbury, N. W., and Yan, H. (2012). Interenzyme substrate diffusion for an enzyme cascade organized on spatially addressable DNA nanostructures. J. Am. Chem. Soc. 134, 5516-5519. doi: 10.1021/ja300897h

Giessen, T. W., and Silver, P. A. (2016). A catalytic nanoreactor based on in vivo encapsulation of multiple enzymes in an engineered protein nanocompartment. ChemBioChem 17, 1931-1935. doi: 10.1002/cbic.201600431

Giessen, T. W., and Silver, P. A. (2017). Widespread distribution of encapsulin nanocompartments reveals functional diversity. Nat. Microbiol. 2:17029. doi: 10.1038/nmicrobiol.2017.29

Gill, S., Catchpole, R., and Forterre, P. (2018). Extracellular membrane vesicles in the three domains of life and beyond. FEMS Microbiol. Rev. 43, 273-303. doi: 10.1093/femsre/fuy042

Ginet, N., Pardoux, R., Adryanczyk, G., Garcia, D., Brutesco, C., and Pignol, D. (2011). Single-step production of a recyclable nanobiocatalyst for organophosphate pesticides biodegradation using functionalized bacterial magnetosomes. PLoS One 6:e21442. doi: 10.1371/journal.pone.0021442

Goldsmith, M., and Tawfik, D. S. (2012). Directed enzyme evolution: beyond the low-hanging fruit. Curr. Opin. Struct. Biol. 22, 406-412. doi: 10.1016/j.sbi.2012. 03.010

Gonzalez-Miro, M., Chen, S., Gonzaga, Z. J., Evert, B., Wibowo, D., and Rehm, B. H. A. (2019). Polyester as antigen carrier toward particulate vaccines. Biomacromolecules 20, 3213-3232. doi: 10.1021/acs.biomac.9b0 0509

Gonzalez-Miro, M., Radecker, A.-M., Rodríguez-Noda, L. M., Fariñas-Medina, M., Zayas-Vignier, C., Hernández-Cedeño, M., et al. (2018a). Design and biological assembly of polyester beads displaying pneumococcal antigens as particulate vaccine. ACS Biomater. Sci. Eng. 4, 3413-3424. doi: 10.1021/acsbiomaterials. $8 \mathrm{~b} 00579$

Gonzalez-Miro, M., Rodríguez-Noda, L. M., Fariñas-Medina, M., Cedré-Marrero, B., Madariaga-Zarza, S., Zayas-Vignier, C., et al. (2018b). Bioengineered polyester beads co-displaying protein and carbohydrate-based antigens induce protective immunity against bacterial infection. Sci. Rep. 8:1888. doi: 10.1038/ s41598-018-20205-7

Grage, K., Jahns, A. C., Parlane, N., Palanisamy, R., Rasiah, I. A., Atwood, J. A., et al. (2009). Bacterial polyhydroxyalkanoate granules: biogenesis, structure, and potential use as nano-/micro-beads in biotechnological and biomedical applications. Biomacromolecules 10, 660-669. doi: 10.1021/bm801394s

Greening, D. W., and Simpson, R. J. (2018). Understanding extracellular vesicle diversity-current status. Expert Rev. Proteomics 15, 887-910. doi: 10.1080/ 14789450.2018 .1537788

Guisan, J. M. (2006). Immobilization of Enzymes and Cells. Berlin: Springer Science \& Business Media. doi: 10.1007/978-1-62703-550-7

Hartmeier, W. (2012). Immobilized Biocatalysts: An Introduction. Berlin: Springer Science \& Business Media. doi: 10.1007/978-3-642-73364-2

Hay, I. D., Du, J., Burr, N., and Rehm, B. H. (2015). Bioengineering of bacteria to assemble custom-made polyester affinity resins. Appl. Environ. Microbiol. 81, 282-291. doi: 10.1128/AEM.02595-14

Hay, I. D., Hooks, D. O., and Rehm, B. H. (2014). "Use of bacterial polyhydroxyalkanoates in protein display technologies," in Hydrocarbon and 
Lipid Microbiology Protocols, eds T. J. McGenity, K. N. Timmis, and B. N. Fernandez, (Berlin: Springer), 71-86. doi: 10.1007/8623_2014_34

Hess, G. T., Cragnolini, J. J., Popp, M. W., Allen, M. A., Dougan, S. K., Spooner, E., et al. (2012). M13 bacteriophage display framework that allows sortasemediated modification of surface-accessible phage proteins. Bioconjug. Chem. 23, 1478-1487. doi: 10.1021/bc300130z

Homaei, A. A., Sariri, R., Vianello, F., and Stevanato, R. (2013). Enzyme immobilization: an update. J. Chem. Biol. 6, 185-205. doi: 10.1007/s12154-013$0102-9$

Honda, T., Tanaka, T., and Yoshino, T. (2015a). Stoichiometrically controlled immobilization of multiple enzymes on magnetic nanoparticles by the magnetosome display system for efficient cellulose hydrolysis. Biomacromolecules 16, 3863-3868. doi: 10.1021/acs.biomac.5b01174

Honda, T., Yasuda, T., Tanaka, T., Hagiwara, K., Arai, T., and Yoshino, T. (2015b). Functional expression of full-Length TrkA in the prokaryotic host Magnetospirillum magneticum AMB-1 by using a magnetosome display system. Appl. Environ. Microbiol. 81, 1472-1476. doi: 10.1128/aem.03112-14

Hooks, D. O., Blatchford, P. A., and Rehm, B. H. A. (2013). Bioengineering of bacterial polymer inclusions catalyzing the synthesis of $\mathrm{N}$-acetylneuraminic acid. Appl. Environ. Microbiol. 79, 3116-3121. doi: 10.1128/aem.03947-12

Hooks, D. O., and Rehm, B. H. (2015). Surface display of highly-stable Desulfovibrio vulgaris carbonic anhydrase on polyester beads for $\mathrm{CO} 2$ capture. Biotechnol. Lett. 37, 1415-1420. doi: 10.1007/s10529-015-1803-7

Hooks, D. O., Venning-Slater, M., Du, J., and Rehm, B. (2014). Polyhydroyxalkanoate synthase fusions as a strategy for oriented enzyme immobilisation. Molecules 19, 8629-8643. doi: 10.3390/molecules19068629

Hsia, Y., Bale, J. B., Gonen, S., Shi, D., Sheffler, W., Fong, K. K., et al. (2016). Design of a hyperstable 60-subunit protein icosahedron. Nature 535, 136-139. doi: $10.1038 /$ nature 18010

Hwang, E. T., and Lee, S. (2019). Multienzymatic cascade reactions via enzyme complex by immobilization. ACS Catal. 9, 4402-4425. doi: 10.1021/acscatal. $8 \mathrm{~b} 04921$

Islam, T., Peng, C., and Ali, I. (2018). Morphological and cellular diversity of magnetotactic bacteria: a review. J. Basic Microbiol. 58, 378-389. doi: 10.1002/ jobm. 201700383

Jacob, J. J., and Suthindhiran, K. (2016). Magnetotactic bacteria and magnetosomes - Scope and challenges. Mater. Sci. Eng. C 68, 919-928. doi: $10.1016 /$ j.msec.2016.07.049

Jahns, A. C., and Rehm, B. H. (2015). Immobilization of active lipase B from Candida antarctica on the surface of polyhydroxyalkanoate inclusions. Biotechnol. Lett. 37, 831-835. doi: 10.1007/s10529-014-1735-7

Jakobson, C. M., Chen, Y., Slininger, M. F., Valdivia, E., Kim, E. Y., and TullmanErcek, D. (2016). Tuning the catalytic activity of subcellular nanoreactors. J. Mol. Biol. 428, 2989-2996. doi: 10.1016/j.jmb.2016.07.006

Jakobson, C. M., Slininger Lee, M. F., and Tullman-Ercek, D. (2017). De novo design of signal sequences to localize cargo to the 1, 2-propanediol utilization microcompartment. Protein Sci. 26, 1086-1092. doi: 10.1002/pro. 3144

Janitzek, C. M., Peabody, J., Thrane, S., Carlsen, P. H., Theander, T. G., Salanti, A., et al. (2019). A proof-of-concept study for the design of a VLP-based combinatorial HPV and placental malaria vaccine. Sci. Rep. 9:5260. doi: 10. 1038/s41598-019-41522-5

Jegannathan, K. R., and Nielsen, P. H. (2013). Environmental assessment of enzyme use in industrial production - a literature review. J. Clean. Prod. 42, 228-240. doi: 10.1016/j.jclepro.2012.11.005

Jia, J., Peng, X., Qi, W., Su, R., and He, Z. (2017). Effects of macromolecular crowding on alkaline phosphatase unfolding, conformation and stability. Int. J. Biol. Macromol. 101, 373-382. doi: 10.1016/j.ijbiomac.2017.03.113

Jordan, P. C., Patterson, D. P., Saboda, K. N., Edwards, E. J., Miettinen, H. M., Basu, G., et al. (2016). Self-assembling biomolecular catalysts for hydrogen production. Nat. Chem. 8, 179-185. doi: 10.1038/nchem.2416

Keshavarz, T., and Roy, I. (2010). Polyhydroxyalkanoates: bioplastics with a green agenda. Curr. Opin. Microbiol. 13, 321-326. doi: 10.1016/j.mib.2010. 02.006

Kikkawa, Y., Narike, M., Hiraishi, T., Kanesato, M., Sudesh, K., Doi, Y., et al. (2005). Organization of polyhydroxyalkanoate synthase for in vitro polymerization as revealed by atomic force microscopy. Macromol. Biosci. 5, 929-935. doi: 10.1002/mabi.200500115
Kim, J., Kim, Y. J., Choi, S. Y., Lee, S. Y., and Kim, K. J. (2017). Crystal structure of Ralstonia eutropha polyhydroxyalkanoate synthase C-terminal domain and reaction mechanisms. Biotechnol. J. 12:1600648. doi: 10.1002/biot.201600648

Kim, Y. J., Choi, S. Y., Kim, J., Jin, K. S., Lee, S. Y., and Kim, K. J. (2017). Structure and function of the N-terminal domain of Ralstonia eutropha polyhydroxyalkanoate synthase, and the proposed structure and mechanisms of the whole enzyme. Biotechnol. J. 12:1600649. doi: 10.1002/biot.201600649

King, N. P., Bale, J. B., Sheffler, W., Mcnamara, D. E., Gonen, S., Gonen, T., et al. (2014). Accurate design of co-assembling multi-component protein nanomaterials. Nature 510, 103-108. doi: 10.1038/nature13404

Koch, C., Wabbel, K., Eber, F. J., Krolla-Sidenstein, P., Azucena, C., Gliemann, H., et al. (2015). Modified TMV particles as beneficial scaffolds to present sensor enzymes. Front. Plant Sci. 6:1137. doi: 10.3389/fpls.2015.01137

Koller, M. (2018). Biodegradable and biocompatible polyhydroxy-alkanoates (PHA): auspicious microbial macromolecules for pharmaceutical and therapeutic applications. Molecules 23:362. doi: 10.3390/molecules23020362

Koller, M., Salerno, A., Dias, M., Reiterer, A., and Braunegg, G. (2010). Modern biotechnological polymer synthesis: a review. Food Technol. Biotechnol. 48, 255-269. doi: 10.1016/j.biombioe.2010.10.008

Krauss, U., Jäger, V. D., Diener, M., Pohl, M., and Jaeger, K.-E. (2017). Catalyticallyactive inclusion bodies-carrier-free protein immobilizates for application in biotechnology and biomedicine. J. Biotechnol. 258, 136-147. doi: 10.1016/j. jbiotec.2017.04.033

Lau, Y. H., Giessen, T. W., Altenburg, W. J., and Silver, P. A. (2018). Prokaryotic nanocompartments form synthetic organelles in a eukaryote. Nat. Commun. 9:1311. doi: 10.1038/s41467-018-03768-x

Lee, E. J. (2018). Recent advances in protein-based nanoparticles. Korean J. Chem. Eng. 35, 1765-1778. doi: 10.1007/s11814-018-0102-0

Lee, H.-J. (2019). Microbe-host communication by small RNAs in extracellular vesicles: vehicles for transkingdom RNA transportation. Int. J. Mol. Sci. 20:1487. doi: 10.3390/ijms20061487

Lee, M. J., Brown, I. R., Juodeikis, R., Frank, S., and Warren, M. J. (2016). Employing bacterial microcompartment technology to engineer a shell-free enzyme-aggregate for enhanced 1, 2-propanediol production in Escherichia coli. Metab. Eng. 36, 48-56. doi: 10.1016/j.ymben.2016.02.007

Lee, S. L., O’ Connor, T. F., Yang, X., Cruz, C. N., Chatterjee, S., Madurawe, R. D., et al. (2015). Modernizing pharmaceutical manufacturing: from batch to continuous production. J. Pharm. Innov. 10, 191-199. doi: 10.1007/s12247015-9215-8

Lee, S. Y. (1996). High cell-density culture of Escherichia coli. Trends Biotechnol. 14, 98-105. doi: 10.1016/0167-7799(96)80930-9

Li, H., Zheng, G., and Zhu, S. (2018). Construction of an organelle-like nanodevice via supramolecular self-assembly for robust biocatalysts. Microb. Cell Fact. 17:26. doi: 10.1186/s12934-018-0873-3

Li, R., Yang, J., Xiao, Y., and Long, L. (2019). In vivo immobilization of an organophosphorus hydrolyzing enzyme on bacterial polyhydroxyalkanoate nano-granules. Microb. Cell Fact. 18:166. doi: 10.1186/s12934-019-1201-2

Lohße, A., Borg, S., Raschdorf, O., Kolinko, I., Tompa, É., Pósfai, M., et al. (2014). Genetic dissection of the mamAB and mms6 operons reveals a gene set essential for magnetosome biogenesis in Magnetospirillum gryphiswaldense. J. Bacteriol. 196, 2658-2669. doi: 10.1128/jb.01716- 14

Longatti, A., Schindler, C., Collinson, A., Jenkinson, L., Matthews, C., Fitzpatrick, L., et al. (2018). High affinity single-chain variable fragments are specific and versatile targeting motifs for extracellular vesicles. Nanoscale 10, 14230-14244. doi: $10.1039 / \mathrm{c} 8 \mathrm{nr} 03970 \mathrm{~d}$

Mathuriya, A. S., and Yakhmi, J. (2017). "Polyhydroxyalkanoates: biodegradable plastics and their applications," in Handbook of Ecomaterials, eds L. M. T. Martínez, O. V. Kharissova, and B. I. Kharisov, (Cham: Springer International Publishing AG), 1-29. doi: 10.1007/978-3-319-48281-1_84-1

Meng, D.-C., Shen, R., Yao, H., Chen, J.-C., Wu, Q., and Chen, G.-Q. (2014). Engineering the diversity of polyesters. Curr. Opin. Biotechnol. 29, 24-33. doi: 10.1016/j.copbio.2014.02.013

Mickoleit, F., and Schüler, D. (2018). Generation of multifunctional magnetic nanoparticles with amplified catalytic activities by genetic expression of enzyme arrays on bacterial magnetosomes. Adv. Biosyst. 2:1700109. doi: 10.1002/adbi. 201700109

Minton, A. P. (2000). Effect of a concentrated "inert" macromolecular cosolute on the stability of a globular protein with respect to denaturation by heat 
and by chaotropes: a statistical-thermodynamic model. Biophys. J. 78, 101-109. doi: 10.1016/S0006-3495(00)76576-3

Mohamad, N. R., Marzuki, N. H. C., Buang, N. A., Huyop, F., and Wahab, R. A. (2015). An overview of technologies for immobilization of enzymes and surface analysis techniques for immobilized enzymes. Biotechnol. Biotechnol. Equip. 29, 205-220. doi: 10.1080/13102818.2015.1008192

Mohsen, M. O., Zha, L., Cabral-Miranda, G., and Bachmann, M. F. (2017). Major findings and recent advances in virus-like particle (VLP)-based vaccines. Semin. Immunol. 34, 123-132. doi: 10.1016/j.smim.2017.08.014

Moldes, C., Farinós, G. P., De Eugenio, L. I., García, P., García, J. L., Ortego, F., et al. (2006). New tool for spreading proteins to the environment: crylAb toxin immobilized to bioplastics. Appl. Microbiol. Biotechnol. 72, 88-93. doi: 10.1007/s00253-005-0257-6

Moldes, C., García, P., García, J. L., and Prieto, M. A. (2004). In vivo immobilization of fusion proteins on bioplastics by the novel tag BioF. Appl. Environ. Microbiol. 70, 3205-3212. doi: 10.1128/AEM.70.6.3205-3212.2004

Moradali, M. F., and Rehm, B. H. A. (2020). Bacterial biopolymers: from pathogenesis to advanced materials. Nat. Rev. Microbiol. doi: 10.1038/s41579019-0313-3

Nguyen, H. H., and Kim, M. (2017). An overview of techniques in enzyme immobilization. Appl. Sci. Converg. Technol. 26, 157-163. doi: 10.5757/ASCT. 2017.26.6.157

Nguyen, T., Pellegrin, B., Bernard, C., Gu, X., Gorham, J. M., Stutzman, P., et al. (2011). Fate of nanoparticles during life cycle of polymer nanocomposites. J. Phys. Conf. Ser. 304:012060. doi: 10.1088/1742-6596/304/1/012060

Ogura, K., and Rehm, B. H. A. (2019). Alginate encapsulation of bioengineered protein-coated polyhydroxybutyrate particles: a new platform for multifunctional composite materials. Adv. Funct. Mater. 29:1901893. doi: 10.1002/adfm.201901893

Ohno, S.-I., Takanashi, M., Sudo, K., Ueda, S., Ishikawa, A., Matsuyama, N., et al. (2013). Systemically injected exosomes targeted to EGFR deliver antitumor microRNA to breast cancer cells. Mol. Ther. 21, 185-191. doi: 10.1038/mt.2012. 180

Ong, S. Y., Chee, J. Y., and Sudesh, K. (2017). Degradation of polyhydroxyalkanoate (PHA): a review. J. Sib. Fed. Univ. Biol. 10, 211-225. doi: 10.17516/1997-13890024

Palomo, J. M., and Guisan, J. M. (2012). "Different strategies for hyperactivation of lipase biocatalysts," in Lipases and Phospholipases: Methods and Protocols, ed. G. Sandoval, (Totowa, NJ: Humana Press), 329-341. doi: 10.1007/978-1-61779600-5_20

Park, M., Sun, Q., Liu, F., Delisa, M. P., and Chen, W. (2014). Positional assembly of enzymes on bacterial outer membrane vesicles for cascade reactions. PLoS One 9:e97103. doi: 10.1371/journal.pone.0097103

Parlane, N. A., Chen, S., Jones, G. J., Vordermeier, H. M., Wedlock, D. N., Rehm, B. H. A., et al. (2016a). Display of antigens on polyester inclusions lowers the antigen concentration required for a bovine tuberculosis skin test. Clin. Vaccine Immunol. 23, 19-26. doi: 10.1128/cvi.00462-15

Parlane, N. A., Gupta, S. K., Rubio-Reyes, P., Chen, S., Gonzalez-Miro, M., Wedlock, D. N., et al. (2016b). Self-assembled protein-coated polyhydroxyalkanoate beads: properties and biomedical applications. ACS Biomater. Sci. Eng. 3, 3043-3057. doi: 10.1021/acsbiomaterials.6b0 0355

Patterson, D., Edwards, E., and Douglas, T. (2015). Hybrid nanoreactors: coupling enzymes and small-molecule catalysts within virus-like particles. Isr. J. Chem. 55, 96-101. doi: 10.1002/ijch.201400092

Peoples, O. P., and Sinskey, A. J. (1989a). Poly-beta-hydroxybutyrate biosynthesis in Alcaligenes eutrophus H16. Characterization of the genes encoding beta-ketothiolase and acetoacetyl-CoA reductase. J. Biol. Chem. 264, 15293-15297.

Peoples, O. P., and Sinskey, A. J. (1989b). Poly-beta-hydroxybutyrate (PHB) biosynthesis in Alcaligenes eutrophus H16. Identification and characterization of the PHB polymerase gene (phbC). J. Biol. Chem. 264, 15298-15303.

Peschke, T., Skoupi, M., Burgahn, T., Gallus, S., Ahmed, I., Rabe, K. S., et al. (2017). Self-immobilizing fusion enzymes for compartmentalized biocatalysis. ACS Catal. 7, 7866-7872. doi: 10.1021/acscatal.7b02230

Philip, S., Keshavarz, T., and Roy, I. (2007). Polyhydroxyalkanoates: biodegradable polymers with a range of applications. J. Chem. Technol. Biotechnol. 82, 233247. doi: $10.1002 /$ jctb.1667
Pieper-Fürst, U., Madkour, M. H., Mayer, F., and Steinbüchel, A. (1994). Purification and characterization of a 14-kilodalton protein that is bound to the surface of polyhydroxyalkanoic acid granules in Rhodococcus ruber. J. Bacteriol. 176, 4328-4337. doi: 10.1128/jb.176.14.4328-4337.1994

Raeeszadeh-Sarmazdeh, M., Hartzell, E., Price, J. V., and Chen, W. (2016). Protein nanoparticles as multifunctional biocatalysts and health assessment sensors. Curr. Opin. Chem. Eng. 13, 109-118. doi: 10.1016/j.coche.2016.08.016

Ran, G., Tan, D., Dai, W., Zhu, X., Zhao, J., Ma, Q., et al. (2017). Immobilization of alkaline polygalacturonate lyase from Bacillus subtilis on the surface of bacterial polyhydroxyalkanoate nano-granules. Appl. Microbiol. Biotechnol. 101, 3247-3258. doi: 10.1007/s00253-016-8085-4

Ran, G., Tan, D., Zhao, J., Fan, F., Zhang, Q., Wu, X., et al. (2019). Functionalized polyhydroxyalkanoate nano-beads as a stable biocatalyst for cost-effective production of the rare sugar D-allulose. Bioresour. Technol. 289:121673. doi: 10.1016/j.biortech.2019.121673

Raposo, G., and Stoorvogel, W. (2013). Extracellular vesicles: exosomes, microvesicles, and friends. J. Cell Biol. 200, 373-383. doi: 10.1083/jcb. 201211138

Rasiah, I. A., and Rehm, B. H. (2009). One-step production of immobilized $\alpha$-amylase in recombinant Escherichia coli. Appl. Environ. Microbiol. 75, 20122016. doi: 10.1128/AEM.02782-08

Rehm, B. H. (2003). Polyester synthases: natural catalysts for plastics. Biochem. J. 376, 15-33. doi: 10.1042/BJ20031254

Rehm, B. H. (2007). Biogenesis of microbial polyhydroxyalkanoate granules: a platform technology for the production of tailor-made bioparticles. Curr. Issues Mol. Biol. 9, 41-62. doi: 10.21775/cimb.009.041

Rehm, B. H. (2010). Bacterial polymers: biosynthesis, modifications and applications. Nat. Rev. Microbiol. 8, 578-592. doi: 10.1038/nrmicro2354

Rehm, F. B., Chen, S., and Rehm, B. (2016). Enzyme engineering for in situ immobilization. Molecules 21:E1370. doi: 10.3390/molecules21101370

Rehm, F. B., Chen, S., and Rehm, B. H. (2018). Bioengineering toward direct production of immobilized enzymes: a paradigm shift in biocatalyst design. Bioengineered 9, 6-11. doi: 10.1080/21655979.2017.1325040

Ren, E., Lei, Z., Wang, J., Zhang, Y., and Liu, G. (2018). Magnetosome modification: from bio-nano engineering toward nanomedicine. Adv. Ther. 1:1800080. doi: 10.1002/adtp.201800080

Ren, S., Li, C., Jiao, X., Jia, S., Jiang, Y., Bilal, M., et al. (2019). Recent progress in multienzymes co-immobilization and multienzyme system applications. Chem. Eng. J. 373, 1254-1278. doi: 10.1016/j.cej.2019.05.141

Robins, K. J., Hooks, D. O., Rehm, B. H., and Ackerley, D. F. (2013). Escherichia coli NemA is an efficient chromate reductase that can be biologically immobilized to provide a cell free system for remediation of hexavalent chromium. PLoS One 8:e59200. doi: 10.1371/journal.pone.0059200

Robinson, P. K. (2015). Enzymes: principles and biotechnological applications. Essays Biochem. 59, 1-41. doi: 10.1042/bse0590001

Rodrigues, R. C., Ortiz, C., Berenguer-Murcia, Á., Torres, R., and FernándezLafuente, R. (2013). Modifying enzyme activity and selectivity by immobilization. Chem. Soc. Rev. 42, 6290-6307. doi: 10.1039/C2CS35231A

Ross, G., Ross, S., and Tighe, B. J. (2017). "Bioplastics: new routes, new products," in Brydson's Plastics Materials, ed. M. Gilbert, (Amsterdam: Elsevier), 631-652. doi: 10.1016/B978-0-323-35824-8.00023-2

Rubio-Reyes, P., Parlane, N. A., Wedlock, D. N., and Rehm, B. H. (2016). Immunogenicity of antigens from Mycobacterium tuberculosis self-assembled as particulate vaccines. Int. J. Med. Microbiol. 306, 624-632. doi: 10.1016/j.ijmm. 2016.10.002

Schmid-Dannert, C., and López-Gallego, F. (2019). Advances and opportunities for the design of self-sufficient and spatially organized cell-free biocatalytic systems. Curr. Opin. Chem. Biol. 49, 97-104. doi: 10.1016/j.cbpa.2018.11.021

Schoonen, L., Maassen, S., Nolte, R. J., and Van Hest, J. C. (2017) Stabilization of a virus-like particle and its application as a nanoreactor at physiological conditions. Biomacromolecules 18, 3492-3497. doi: 10.1021/acs. biomac.7b00640

Schwarz, B., Uchida, M., and Douglas, T. (2017). "Biomedical and catalytic opportunities of virus-like particles in nanotechnology," in Advances in Virus Research, eds M. Kielian, T. C. Mettenleiter, and M. J. Roossinck, (Cambridge, MA: Academic Press), 1-60. doi: 10.1016/bs.aivir.2016.09.002

Seo, H. M., Kim, J. H., Jeon, J. M., Song, H. S., Bhatia, S. K., Sathiyanarayanan, G., et al. (2016). In situ immobilization of lysine decarboxylase on a biopolymer 
by fusion with phasin: immobilization of CadA on intracellular PHA. Process Biochem. 51, 1413-1419. doi: 10.1016/j.procbio.2016.07.019

Sharma, B., Dangi, A. K., and Shukla, P. (2018). Contemporary enzyme based technologies for bioremediation: a review. J. Environ. Manage. 210, 10-22. doi: 10.1016/j.jenvman.2017.12.075

Sletten, E. M., and Bertozzi, C. R. (2009). Bioorthogonal chemistry: fishing for selectivity in a sea of functionality. Angew Chem. Int. Ed. 48, 6974-6998. doi: 10.1002/anie.200900942

Stanley, S. (2014). Biological nanoparticles and their influence on organisms. Curr. Opin. Biotechnol. 28, 69-74. doi: 10.1016/j.copbio.2013.11.014

Steinmann, B., Christmann, A., Heiseler, T., Fritz, J., and Kolmar, H. (2010). In vivo enzyme immobilization by inclusion body display. Appl. Environ. Microbiol. 76, 5563-5569. doi: 10.1128/AEM.00612-10

Su, F.-H., Tabañag, I. D. F., Wu, C.-Y., and Tsai, S.-L. (2017). Decorating outer membrane vesicles with organophosphorus hydrolase and cellulose binding domain for organophosphate pesticide degradation. Chem. Eng. J. 308, 1-7. doi: 10.1016/j.cej.2016.09.045

Sugamata, Y., Uchiyama, R., Honda, T., Tanaka, T., Matsunaga, T., and Yoshino, T. (2013). Functional expression of thyroid-stimulating hormone receptor on nano-sized bacterial magnetic particles in Magnetospirillum magneticum AMB-1. Int. J. Mol. Sci. 14, 14426-14438. doi: 10.3390/ijms140714426

Taguchi, S., and Doi, Y. (2004). Evolution of polyhydroxyalkanoate (PHA) production system by "enzyme evolution": successful case studies of directed evolution. Macromol. Biosci. 4, 145-156. doi: 10.1002/mabi.200300111

Tamborini, L., Fernandes, P., Paradisi, F., and Molinari, F. (2018). Flow bioreactors as complementary tools for biocatalytic process intensification. Trends Biotechnol. 36, 73-88. doi: 10.1016/j.tibtech.2017.09.005

Tan, D., Zhao, J.-P., Ran, G.-Q., Zhu, X.-L., Ding, Y., and Lu, X.-Y. (2019). Highly efficient biocatalytic synthesis of 1-DOPA using in situ immobilized Verrucomicrobium spinosum tyrosinase on polyhydroxyalkanoate nanogranules. Appl. Microbiol. Biotechnol. 103, 5663-5678. doi: 10.1007/s00253019-09851-7

Tan, M., and Jiang, X. (2017). Recent advancements in combination subunit vaccine development. Hum. Vaccin. Immunother. 13, 180-185. doi: 10.1080/ 21645515.2016.1229719

Tarazona, N. A., Machatschek, R., Schulz, B., Prieto, M. A., and Lendlein, A. (2019). Molecular insights into the physical adsorption of amphiphilic protein PhaF onto copolyester surfaces. Biomacromolecules 20, 3242-3252. doi: 10.1021/acs. biomac.9b00069

Tetter, S., and Hilvert, D. (2017). Enzyme encapsulation by a ferritin cage. Angew. Chem. Int. Ed. 56, 14933-14936. doi: 10.1002/anie.201708530

Thompson, M. P., Peñafiel, I., Cosgrove, S. C., and Turner, N. J. (2018). Biocatalysis using immobilized enzymes in continuous flow for the synthesis of fine chemicals. Org. Process Res. Dev. 23, 9-18. doi: 10.1021/acs.oprd.8b00305

Thoniyot, P., Tan, M. J., Karim, A. A., Young, D. J., and Loh, X. J. (2015). Nanoparticle-hydrogel composites: concept, design, and applications of these promising, multi-functional materials. Adv. Sci. 2:1400010. doi: 10.1002/advs. 201400010

Torbensen, K., Patel, A., Anne, A., Chovin, A., Demaille, C., Bataille, L., et al. (2019). Immuno-based molecular scaffolding of glucose dehydrogenase and ferrocene mediator on $\mathrm{fd}$ viral particles yields enhanced bioelectrocatalysis. ACS Catal. 9, 5783-5796. doi: 10.1021/acscatal.9b01263

Truppo, M. D. (2017). Biocatalysis in the pharmaceutical industry: the need for speed. ACS Med. Chem. Lett. 8, 476-480. doi: 10.1021/acsmedchemlett.7b00114

Uebe, R., and Schüler, D. (2016). Magnetosome biogenesis in magnetotactic bacteria. Nat. Rev. Microbiol. 14, 621-637. doi: 10.1038/nrmicro.2016.99

van den Biggelaar, L., Soumillion, P., and Debecker, D. P. (2019). Biocatalytic transamination in a monolithic flow reactor: improving enzyme grafting for enhanced performance. RSC Adv. 9, 18538-18546. doi: 10.1039/c9ra02433f

van der Pol, E., Böing, A. N., Harrison, P., Sturk, A., and Nieuwland, R. (2012). Classification, functions, and clinical relevance of extracellular vesicles. Pharmacol. Rev. 64, 676-705. doi: 10.1124/pr.112.005983

Wagner, H. J., Capitain, C. C., Richter, K., Nessling, M., and Mampel, J. (2017). Engineering bacterial microcompartments with heterologous enzyme cargos. Eng. Life Sci. 17, 36-46. doi: 10.1002/elsc.201600107

Wieczorek, R., Pries, A., Steinbüchel, A., and Mayer, F. (1995). Analysis of a 24kilodalton protein associated with the polyhydroxyalkanoic acid granules in Alcaligenes eutrophus. J. Bacteriol. 177, 2425-2435. doi: 10.1128/jb.177.9.24252435.1995
Wilkerson, J. W., Yang, S.-O., Funk, P. J., Stanley, S. K., and Bundy, B. C. (2018). Nanoreactors: strategies to encapsulate enzyme biocatalysts in virus-like particles. New Biotechnol. 44, 59-63. doi: 10.1016/j.nbt.2018. 04.003

Wittenborn, E. C., Jost, M., Wei, Y., Stubbe, J., and Drennan, C. L. (2016). Structure of the catalytic domain of the class I polyhydroxybutyrate synthase from Cupriavidus necator. J. Biol. Chem. 291, 25264-25277. doi: 10.1074/jbc. M116.756833

Wong, J. X., Gonzalez-Miro, M., Sutherland-Smith, A. J., and Rehm, B. H. A. (2020). Covalent functionalization of bioengineered polyhydroxyalkanoate spheres directed by specific protein-protein interactions. Front. Bioeng. Biotechnol. 8:44. doi: 10.3389/fbioe.2020.00044

Wong, J. X., and Rehm, B. H. (2018). Design of modular polyhydroxyalkanoate scaffolds for protein immobilization by directed ligation. Biomacromolecules 19 , 4098-4112. doi: 10.1021/acs.biomac.8b01093

Xiang, Z., Yang, X., Xu, J., Lai, W., Wang, Z., Hu, Z., et al. (2017). Tumor detection using magnetosome nanoparticles functionalized with a newly screened EGFR/HER2 targeting peptide. Biomaterials 115, 53-64. doi: 10.1016/ j.biomaterials.2016.11.022

Xu, Y., Jiang, S., Simmons, C. R., Narayanan, R. P., Zhang, F., Aziz, A.-M., et al. (2019). Tunable nanoscale cages from self-assembling DNA and protein building blocks. ACS Nano 13, 3545-3554. doi: 10.1021/acsnano.8b09798

Yan, L., Da, H., Zhang, S., López, V. M., and Wang, W. (2017). Bacterial magnetosome and its potential application. Microbiol. Res. 203, 19-28. doi: 10.1016/j.micres.2017.06.005

Yang, Q., Wang, B., Zhang, Z., Lou, D., Tan, J., and Zhu, L. (2017). The effects of macromolecular crowding and surface charge on the properties of an immobilized enzyme: activity, thermal stability, catalytic efficiency and reusability. RSC Adv. 7, 38028-38036. doi: 10.1039/c7ra06544b

Yang, T. H., Kwon, M.-A., Lee, J. Y., Choi, J.-E., Oh, J. Y., and Song, J. K. (2015). In situ immobilized lipase on the surface of intracellular polyhydroxybutyrate granules: preparation, characterization, and its promising use for the synthesis of fatty acid alkyl esters. Appl. Biochem. Biotechnol. 177, 1553-1564. doi: 10. 1007/s12010-015-1836-3

Zaak, H., Siar, E.-H., Kornecki, J. F., Fernandez-Lopez, L., Pedrero, S. G., Virgen-Ortíz, J. J., et al. (2017). Effect of immobilization rate and enzyme crowding on enzyme stability under different conditions. The case of lipase from Thermomyces lanuginosus immobilized on octyl agarose beads. Process Biochem. 56, 117-123. doi: 10.1016/j.procbio.2017.02.024

Zakeri, B., Fierer, J. O., Celik, E., Chittock, E. C., Schwarz-Linek, U., Moy, V. T., et al. (2012). Peptide tag forming a rapid covalent bond to a protein, through engineering a bacterial adhesin. Proc. Natl. Acad. Sci. U.S.A. 109, E690-E697. doi: $10.1073 /$ pnas. 1115485109

Zdarta, J., Meyer, A., Jesionowski, T., and Pinelo, M. (2018). A general overview of support materials for enzyme immobilization: characteristics, properties, practical utility. Catalysts 8:92. doi: 10.3390/catal8020092

Zhang, W. (2014). Nanoparticle aggregation: principles and modeling. Adv. Exp. Med. Biol. 811, 19-43. doi: 10.1007/978-94-017-8739-0_2

Zhao, H., Wei, H., Liu, X., Yao, Z., Xu, M., Wei, D., et al. (2016). Structural insights on PHA binding protein PhaP from Aeromonas hydrophila. Sci. Rep. 6:39424. doi: 10.1038/srep39424

Zhao, H., Yao, Z., Chen, X., Wang, X., and Chen, G. Q. (2017). Modelling of microbial polyhydroxyalkanoate surface binding protein $\mathrm{PhaP}$ for rational mutagenesis. Microb. Biotechnol. 10, 1400-1411. doi: 10.1111/1751-7915.12820

Conflict of Interest: BR is the co-founder and shareholder of PolyBatics Ltd., that commercializes veterinary TB diagnostic products related to the PHA particle technology.

The remaining authors declare that the research was conducted in the absence of any commercial or financial relationships that could be construed as a potential conflict of interest.

Copyright (c) 2020 Wong, Ogura, Chen and Rehm. This is an open-access article distributed under the terms of the Creative Commons Attribution License (CC BY). The use, distribution or reproduction in other forums is permitted, provided the original author(s) and the copyright owner(s) are credited and that the original publication in this journal is cited, in accordance with accepted academic practice. No use, distribution or reproduction is permitted which does not comply with these terms. 